\title{
SPANNING SUBSPACE CONFIGURATIONS
}

\author{
BRENDON RHOADES
}

\begin{abstract}
A spanning configuration in the complex vector space $\mathbb{C}^{k}$ is a sequence $\left(W_{1}, \ldots, W_{r}\right)$ of linear subspaces of $\mathbb{C}^{k}$ such that $W_{1}+\cdots+W_{r}=\mathbb{C}^{k}$. We present the integral cohomology of the moduli space of spanning configurations in $\mathbb{C}^{k}$ corresponding to a given sequence of subspace dimensions. This simultaneously generalizes the classical presentation of the cohomology of partial flag varieties and the more recent presentation of a variety of spanning line configurations defined by the author and Pawlowski. This latter variety of spanning line configurations plays the role of the flag variety for the Haglund-Remmel-Wilson Delta Conjecture of symmetric function theory.
\end{abstract}

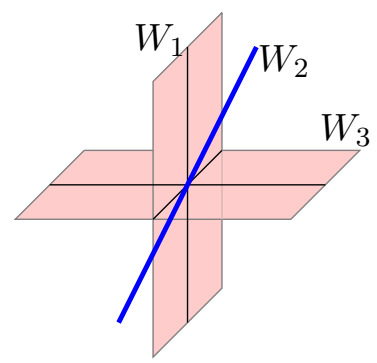

Figure 1. A point in $X_{(2,1,2), 3}$.

\section{INTRODUCTION}

In this paper we compute the cohomology of the moduli space of ordered tuples of subspaces of $\mathbb{C}^{k}$ with a given sequence of dimensions which have full span $\mathbb{C}^{k}$. These varieties generalize classical spaces of Schubert calculus (partial flag varieties) as well as a newer space $X_{n, k}$ of spanning line configurations which has connections to the Delta Conjecture of symmetric function theory [8]. The cohomology rings of these subspace configurations have combinatorics governed by ordered tuples of subsets $\mathcal{I}=\left(I_{1}, \ldots, I_{r}\right)$ of $[k]:=\{1,2, \ldots, k\}$ which satisfy $I_{1} \cup \cdots \cup I_{r}=[k]$.

A finite sequence $\left(W_{1}, \ldots, W_{r}\right)$ of linear subspaces of the $k$-dimensional complex vector space $\mathbb{C}^{k}$ is a spanning configuration if the $W_{i}$ span $\mathbb{C}^{k}$, i.e. $W_{1}+\cdots+W_{r}=\mathbb{C}^{k}$. The dimension vector of $\left(W_{1}, \ldots, W_{r}\right)$ of subspaces of $\mathbb{C}^{k}$ is the sequence of dimensions $\left(\operatorname{dim}\left(W_{1}\right), \ldots, \operatorname{dim}\left(W_{r}\right)\right)$. The following moduli space of spanning configurations is our object of study.

Definition 1.1. Let $k>0$ and let $\alpha=\left(\alpha_{1}, \ldots, \alpha_{r}\right) \in[k]^{r}$. Let $X_{\alpha, k}$ be the moduli space

$$
X_{\alpha, k}:=\left\{\text { all spanning configurations }\left(W_{1}, \ldots, W_{r}\right) \text { in } \mathbb{C}^{k} \text { with dimension vector } \alpha\right\} .
$$

A subspace configuration in $X_{(2,1,2), 3}$ is shown in Figure 1 .

Let $\operatorname{Gr}(d, k)$ be the Grassmannian of all $d$-dimensional subspaces of $\mathbb{C}^{k}$. We have an inclusion

$$
\iota: X_{\alpha, k} \hookrightarrow G r(\alpha, k)
$$

of $X_{\alpha, k}$ into the product of Grassmannians

$$
\operatorname{Gr}(\alpha, k):=\operatorname{Gr}\left(\alpha_{1}, k\right) \times \cdots \times \operatorname{Gr}\left(\alpha_{r}, k\right) .
$$

Key words and phrases. subspace configuration, cohomology. 
This realizes $X_{\alpha, k}$ as a Zariski open subset of $G r(\alpha, k)$. We may therefore view $X_{\alpha, k}$ as either a complex algebraic variety or a smooth complex manifold.

Various old and new moduli spaces arising in Schubert calculus are special cases of the $X_{\alpha, k}$.

- If $\alpha_{i}=k$ for any $i$, then any sequence of subspaces in $\mathbb{C}^{k}$ is a spanning configuration and $X_{\alpha, k}$ equals the Grassmann product $\operatorname{Gr}(\alpha, k)$. Any product of Grassmannians can be obtained in this way.

- If $\alpha=\left(1^{k}\right)$ is a length $k$ sequence of 1 's, then $X_{\left(1^{k}\right), k}$ is homotopy equivalent to the flag variety

$$
\mathcal{F} \ell(k)=\left\{0=V_{0} \subset V_{1} \subset \cdots \subset V_{k}=\mathbb{C}^{k}: \operatorname{dim}\left(V_{i}\right)=i\right\}
$$

of complete flags in $\mathbb{C}^{k}$. To see why this is the case, let $G L_{k}$ be the group of $k \times k$ invertible complex matrices and let $T \subseteq B$ be the subgroups of diagonal and upper triangular matrices. We have a canonical projection

$$
\pi: X_{\left(1^{k}\right), k}=G L_{k} / T \rightarrow G L_{k} / B=\mathcal{F} \ell(k) .
$$

It can be shown that $\pi$ is a fiber bundle with contractible fiber (homeomorphic to the maximal unipotent subgroup of $G L_{k}$ of upper triangular matrices with 1's on the diagonal), so that $\pi$ is a homotopy equivalence.

- More generally, if $\alpha_{1}+\cdots+\alpha_{r}=k$, then $X_{\alpha, k}$ is homotopy equivalent to the partial flag variety

$$
\mathcal{F} \ell(\alpha)=\left\{0=V_{0} \subset V_{1} \subset \cdots \subset V_{r}=\mathbb{C}^{k}: \operatorname{dim}\left(V_{i}\right)=\alpha_{1}+\cdots+\alpha_{i}\right\} .
$$

Any partial flag variety can be obtained in this way.

- Finally, if $\alpha=\left(1^{n}\right)$ for some $k \leq n$, the space $X_{\left(1^{n}\right), k}$ consists of $n$-tuples of lines whose span equals $\mathbb{C}^{k}$. This space of spanning line configurations was introduced by Pawlowski and the author [8] under the name $X_{n, k}$. It was shown that the cohomology $H^{\bullet}\left(X_{\left(1^{n}\right), k} ; \mathbb{Z}\right)$ has rank equal to $k ! \cdot \operatorname{Stir}(n, k)$, where $\operatorname{Stir}(n, k)$ is the Stirling number of the second kind counting set partitions of an $n$-element set into $k$ nonempty blocks.

The space $X_{\left(1^{n}\right), k}$ of the last bullet point has connections to symmetric function theory. We briefly describe this connection here and refer the reader to [1] for details on symmetric function theory.

Given $k \leq n$, the Delta Conjecture of Haglund, Remmel, and Wilson [6] predicts the following identity of formal power series involving infinitely many variables $\mathbf{x}=\left(x_{1}, x_{2}, \ldots\right)$ and two additional parameters $q, t$ :

$$
\Delta_{e_{k-1}}^{\prime} e_{n}=\operatorname{Rise}_{n, k}(\mathbf{x} ; q, t)=\operatorname{Val}_{n, k}(\mathbf{x} ; q, t) .
$$

Here $e_{n}$ is the elementary symmetric function, $\Delta_{e_{k-1}}^{\prime}$ is the primed delta operator attached to $e_{k-1}$, and Rise and Val are certain combinatorially defined formal power series related to lattice paths.

The full Delta Conjecture is open, but it is known when one of the variables $q, t$ is specialized to zero. Combining results in [4, 6, 17, 9, 11] we have

$$
\left.\Delta_{e_{k-1}}^{\prime} e_{n}\right|_{t=0}=\operatorname{Rise}_{n, k}(\mathbf{x} ; q, 0)=\operatorname{Rise}_{n, k}(\mathbf{x} ; 0, q)=\operatorname{Val}_{n, k}(\mathbf{x} ; q, 0)=\operatorname{Val}_{n, k}(\mathbf{x} ; 0, q) .
$$

Let $C_{n, k}(\mathbf{x} ; q)$ be the common symmetric function of Equation (1.8).

Haglund, the author, and Shimozono [7] gave an algebraic model and Pawlowski and the author [8] gave a geometric model for the symmetric function $C_{n, k}(\mathbf{x} ; q)$. Let $\mathbb{Z}\left[\mathbf{x}_{n}\right]:=\mathbb{Z}\left[x_{1}, \ldots, x_{n}\right]$ and $\mathbb{Q}\left[\mathbf{x}_{n}\right]:=\mathbb{Q}\left[x_{1}, \ldots, x_{n}\right]$ be the polynomial rings in $n$ variables over the ring of integers and field of rational numbers. Recall the elementary symmetric polynomial $e_{d}\left(\mathbf{x}_{n}\right)$ and complete homogeneous symmetric polynomial $h_{d}\left(\mathbf{x}_{n}\right)$ of degree $d$ in the variable set $\mathbf{x}_{n}$ :

$$
e_{d}\left(\mathbf{x}_{n}\right):=\sum_{1 \leq i_{1}<\cdots<i_{d} \leq n} x_{i_{1}} \cdots x_{i_{d}}, \quad h_{d}\left(\mathbf{x}_{n}\right):=\sum_{1 \leq i_{1} \leq \cdots \leq i_{d} \leq n} x_{i_{1}} \cdots x_{i_{d}} .
$$


For $k \leq n$, we follow [7, 8] and define the ideals

$$
\begin{aligned}
I_{n, k} & :=\left\langle x_{1}^{k}, x_{2}^{k}, \ldots, x_{n}^{k}, e_{n}\left(\mathbf{x}_{n}\right), e_{n-1}\left(\mathbf{x}_{n}\right), \ldots, e_{n-k+1}\left(\mathbf{x}_{n}\right)\right\rangle \subseteq \mathbb{Z}\left[\mathbf{x}_{n}\right], \\
J_{n, k} & :=\left\langle x_{1}^{k}, x_{2}^{k}, \ldots, x_{n}^{k}, e_{n}\left(\mathbf{x}_{n}\right), e_{n-1}\left(\mathbf{x}_{n}\right), \ldots, e_{n-k+1}\left(\mathbf{x}_{n}\right)\right\rangle \subseteq \mathbb{Q}\left[\mathbf{x}_{n}\right] .
\end{aligned}
$$

Let $R_{n, k}:=\mathbb{Z}\left[\mathbf{x}_{n}\right] / I_{n, k}$ and $S_{n, k}:=\mathbb{Q}\left[\mathbf{x}_{n}\right] / J_{n, k}$ be the corresponding quotient rings.

Let $\mathfrak{S}_{n}$ denote the symmetric group on $n$ letters. We recall the Frobenius image encoding of the isomorphism type of a (graded) $\mathfrak{S}_{n}$-module as a symmetric function.

The irreducible representations of $\mathfrak{S}_{n}$ are in one-to-one correspondence with partitions $\lambda \vdash n$. If $\lambda \vdash n$ is a partition, let $S^{\lambda}$ denote the corresponding irreducible $\mathfrak{S}_{n}$-module. Any finite-dimensional $\mathfrak{S}_{n}$-module $V$ may be expressed as $V \cong \bigoplus_{\lambda \vdash n} m_{\lambda} S^{\lambda}$ for some unique multiplicities $m_{\lambda} \geq 0$. The Frobenius image of $V$ is the symmetric function $\operatorname{Frob}(V):=\sum_{\lambda \vdash n} m_{\lambda} s_{\lambda}$, where $s_{\lambda}$ is the Schur function. Furthermore, if $V=\bigoplus_{d \geq 0} V_{d}$ is a graded $\mathfrak{S}_{n}$-module with each piece $V_{d}$ finite-dimensional and $q$ is a grading variable, the graded Frobenius image is $\operatorname{grFrob}(V ; q):=\sum_{d \geq 0} \operatorname{Frob}\left(V_{d}\right) \cdot q^{d}$.

Theorem 1.2. Let $k \leq n$ be positive integers.

(1) (Pawlowski-R. 8]) The singular cohomology ring $H^{\bullet}\left(X_{\left(1^{n}\right), k} ; \mathbb{Z}\right)$ may be presented as

$$
H^{\bullet}\left(X_{\left(1^{n}\right), k} ; \mathbb{Z}\right)=R_{n, k},
$$

where the variable $x_{i}$ represents the Chern class $c_{1}\left(\ell_{i}^{*}\right) \in H^{2}\left(X_{\left(1^{n}\right)} ; \mathbb{Z}\right)$.

(2) (Haglund-R.-Shimozono [7]) The graded Frobenius image of the graded $\mathfrak{S}_{n}$-modue $S_{n, k}$ is given by

$$
\operatorname{grFrob}\left(S_{n, k} ; q\right)=\left(\operatorname{rev}_{q} \circ \omega\right) C_{n, k}(\mathbf{x} ; q) .
$$

Here $\operatorname{rev}_{q}$ is the operator which reverses the coefficient sequences of polynomials in $q$ and $\omega$ is the symmetric function involution which trades $e_{n}$ and $h_{n}$.

Since the cohomology of $X_{\left(1^{n}\right), k}$ is concentrated in even dimensions, the Universal Coefficient Theorem and the above result imply that

$$
\operatorname{grFrob}\left(H^{\bullet}\left(X_{\left(1^{n}\right), k} ; \mathbb{Q}\right) ; q\right)=\left(\operatorname{rev}_{q} \circ \omega\right) C_{n, k}(\mathbf{x} ; q) .
$$

In this sense, the space $X_{\left(1^{n}\right), k}$ furnishes a geometric model for the Delta Conjecture.

Now consider the spaces $X_{\alpha, k}$ in the case $k=n$. That is, suppose we have a sequence $\alpha=$ $\left(\alpha_{1}, \ldots, \alpha_{r}\right) \in[n]^{r}$ such that $\alpha_{1}+\cdots+\alpha_{r}=n$. In this case, the space $X_{\alpha, n}$ is homotopy equivalent to the partial flag variety $\mathcal{F} \ell(\alpha)$. There is a classical presentation of the cohomology ring $H^{\bullet}(\mathcal{F} \ell(\alpha) ; \mathbb{Z})$ due to Borel. To state it, we introduce some notation.

Let $\alpha=\left(\alpha_{1}, \ldots, \alpha_{r}\right)$ be a length $r$ sequence of positive integers with $\alpha_{1}+\cdots+\alpha_{r}=n$. We break up the list $\mathbf{x}_{n}=\left(x_{1}, \ldots, x_{n}\right)$ of variables into $r$ sublists $\mathbf{x}_{n}^{(1)}, \ldots, \mathbf{x}_{n}^{(r)}$ where

$$
\mathbf{x}_{n}^{(i)}:=\left(x_{\alpha_{1}+\cdots+\alpha_{i-1}+1}, \ldots, x_{\alpha_{1}+\cdots+\alpha_{i-1}+\alpha_{i}}\right)
$$

for $1 \leq i \leq r$. For example, if $\alpha=(2,1,2)$ so that $r=3$ and $n=5$ then

$$
\mathbf{x}_{5}^{(1)}=\left(x_{1}, x_{2}\right), \quad \mathbf{x}_{5}^{(2)}=\left(x_{3}\right), \quad \mathbf{x}_{5}^{(3)}=\left(x_{4}, x_{5}\right) .
$$

Let $\mathfrak{S}_{\alpha}:=\mathfrak{S}_{\alpha_{1}} \times \cdots \times \mathfrak{S}_{\alpha_{r}}$ be the parabolic subgroup of $\mathfrak{S}_{n}$ attached to $\alpha$. The factor $\mathfrak{S}_{\alpha_{i}}$ of $\mathfrak{S}_{\alpha}$ acts on the subscripts of variables in $\mathbf{x}_{n}^{(i)}$. Let $\mathbb{Z}\left[\mathbf{x}_{n}\right]^{\mathfrak{S}_{\alpha}}$ be the ring of polynomials in $\mathbf{x}_{n}$ which are invariant under the action of $\mathfrak{S}_{\alpha}$.

Theorem 1.3. (Borel [2]) Let $\alpha=\left(\alpha_{1}, \ldots, \alpha_{r}\right) \in[n]^{r}$ be a sequence of positive integers with $\alpha_{1}+\cdots+\alpha_{r}=n$. The cohomology ring $H^{\bullet}(\mathcal{F} \ell(\alpha) ; \mathbb{Z})=H^{\bullet}\left(X_{\alpha, k} ; \mathbb{Z}\right)$ may be presented as

$$
H^{\bullet}(\mathcal{F} \ell(\alpha) ; \mathbb{Z})=\left(\mathbb{Z}\left[\mathbf{x}_{n}\right] / I\right)^{\mathfrak{S}_{\alpha}},
$$

where $I \subseteq \mathbb{Z}\left[\mathbf{x}_{n}\right]$ is the ideal generated by the complete homogeneous symmetric polynomials $h_{d}\left(\mathbf{x}_{n}^{(i)}\right)$ where $d>n-\alpha_{i}$ and $1 \leq i \leq r$. 
Our main result presents the cohomology ring $H^{\bullet}\left(X_{\alpha, k} ; \mathbb{Z}\right)$ of $X_{\alpha, k}$ for an arbitrary dimension vector $\alpha \in[k]^{r}$. This simultaneously generalizes Borel's presentation of the cohomology of partial flag varieties in Theorem 1.3 and the geometric model for the Delta Conjecture due to Pawlowski and the author in Theorem 1.2 .

Theorem 1.4. Let $\alpha=\left(\alpha_{1}, \ldots, \alpha_{r}\right) \in[k]^{r}$ be a sequence of positive integers with $\alpha_{1}+\cdots+\alpha_{r}=n$. The singular cohomology ring $H^{\bullet}\left(X_{\alpha, k} ; \mathbb{Z}\right)$ may be presented as

$$
H^{\bullet}\left(X_{\alpha, k} ; \mathbb{Z}\right)=\left(\mathbb{Z}\left[\mathbf{x}_{n}\right] / I_{\alpha, k}\right)^{\mathfrak{S}_{\alpha}},
$$

where $I_{\alpha, k} \subseteq \mathbb{Z}\left[\mathbf{x}_{n}\right]$ is the ideal generated by the complete homogeneous symmetric polynomials $h_{d}\left(\mathbf{x}_{n}^{(i)}\right)$ where $d>n-\alpha_{i}$ and $1 \leq i \leq r$ together with the elementary symmetric polynomials $e_{n}\left(\mathbf{x}_{n}\right), e_{n-1}\left(\mathbf{x}_{n}\right), \ldots, e_{n-k+1}\left(\mathbf{x}_{n}\right)$. Here the variables $\mathbf{x}_{n}^{(i)}$ represent the Chern roots of the tautological vector bundle $W_{i}^{*} \rightarrow X_{\alpha, k}$.

Theorem 1.4 will be proven in Section [5. The $\operatorname{ring} H^{\bullet}\left(X_{\alpha, k} ; \mathbb{Z}\right)$ is a free $\mathbb{Z}$-module and we can describe its rank combinatorially. Given $\alpha=\left(\alpha_{1}, \ldots, \alpha_{r}\right) \in[k]^{r}$, the rank of $H^{\bullet}\left(X_{\alpha, k} ; \mathbb{Z}\right)$ equals the number of 0,1 -matrices $A$ of size $k \times r$ with column sum vector $\alpha$ containing no row of zeros. For example, if $k=3$ and $\alpha=(2,1,2,1)$ a matrix contributing to the rank of $H^{\bullet}\left(X_{(2,1,2,1)} ; \mathbb{Z}\right)$ is

$$
\left(\begin{array}{llll}
0 & 1 & 1 & 0 \\
1 & 0 & 1 & 1 \\
1 & 0 & 0 & 0
\end{array}\right) .
$$

When $\alpha=(d, \ldots, d) \in[k]^{r}$ is a constant vector, the symmetric group $\mathfrak{S}_{r}$ acts on $X_{\alpha, k}$ by subspace permutation:

$$
\pi \cdot\left(W_{1}, \ldots, W_{r}\right):=\left(W_{\pi(1)}, \ldots, W_{\pi(r)}\right)
$$

for $\pi \in \mathfrak{S}_{r}$ and $\left(W_{1}, \ldots, W_{r}\right) \in X_{\alpha, k}$. This induces an action on the rational cohomology $H^{\bullet}\left(X_{\alpha, k} ; \mathbb{Q}\right)$ which coincides with the action of $\mathfrak{S}_{r}$ on the columns of the 0,1-matrices given in the last paragraph.

The proof of Theorem 1.4 is a mixture of classical and modern techniques. We describe the cohomology of $H^{\bullet}\left(X_{\alpha, k} ; \mathbb{Z}\right)$ as a graded group by describing a nonstandard affine paving of the Grassmann product $\operatorname{Gr}(\alpha, k)$ which interacts nicely with the inclusion $X_{\alpha, k} \subseteq G r(\alpha, k)$. This affine paving relies on a linear algebra algorithm combining row and column operations which we call 'mixed reduction' (see Section 4 ). The analysis of the quotient ring $\left(\mathbb{Z}\left[\mathbf{x}_{n}\right] / I_{\alpha, k}\right)^{\mathfrak{S}_{\alpha}}$ relies on the method of orbit harmonics and, in particular, a Demazure character identity which relies on a refinement of the dual Pieri rule due to Haglund, Luoto, Mason, and van Willigenburg [5].

The remainder of the paper is organized as follows. In Section 2 we give background on the combinatorial objects we will be using, Gröbner theory, affine pavings of varieties, and Chern classes. In Section 3 we will use the method of orbit harmonics to analyze the quotient $\mathbb{Z}\left[\mathbf{x}_{n}\right] / I_{\alpha, k}$ (upon extending coefficients to $\mathbb{Q}$ ). In Section 4 we introduce our linear algebra algorithm of mixed reduction and apply it to get a strategic affine paving of $\operatorname{Gr}(\alpha, k)$ which restricts to an affine paving of $X_{\alpha, k}$. In Section 5 we prove Theorem 1.4 and present the cohomology of $X_{\alpha, k}$. We close in Section 6 with some open problems.

\section{BACKGROUND}

2.1. Combinatorics. A (strong) composition is a finite sequence $\alpha=\left(\alpha_{1}, \ldots, \alpha_{r}\right)$ of positive integers. If $\alpha_{1}+\cdots+\alpha_{r}=n$, we say that $\alpha$ is a composition of $n$ and write $|\alpha|=n$. We also say that $\alpha$ has $r$ parts and write $\ell(\alpha)=r$. We will be concerned with compositions $\alpha \in[k]^{r}$ whose parts are bounded above by a constant $k$. 
Let $\alpha=\left(\alpha_{1}, \ldots, \alpha_{r}\right) \in[k]^{r}$ with $|\alpha|=n$ and suppose we have an ordered list of $n$ things (such as variables in $\left(x_{1}, x_{2}, \ldots, x_{n}\right)$ or the columns of an $n$-column matrix read from left to right). For $1 \leq i \leq r$, we will refer to the subset of these things indexed by the set

$$
\left\{\alpha_{1}+\cdots+\alpha_{i-1}+1, \alpha_{1}+\cdots+\alpha_{i-1}+2, \ldots, \alpha_{1}+\cdots+\alpha_{i-1}+\alpha_{i}\right\}
$$

as the $i^{\text {th }}$ batch (with dependence on $\alpha$ understood). For example, if $\alpha=(2,1,2)$ and we have the list of variables $\mathbf{x}_{5}=\left(x_{1}, x_{2}, x_{3}, x_{4}, x_{5}\right)$, the first batch is $\mathbf{x}_{5}^{(1)}=\left(x_{1}, x_{2}\right)$, the second batch is $\mathbf{x}_{5}^{(2)}=\left(x_{3}\right)$, and the third batch is $\mathbf{x}_{5}^{(3)}=\left(x_{4}, x_{5}\right)$.

If $\alpha=\left(\alpha_{1}, \ldots, \alpha_{r}\right) \in[k]^{r}$ with $|\alpha|=n$, let $\mathfrak{S}_{\alpha}:=\mathfrak{S}_{\alpha_{1}} \times \cdots \mathfrak{S}_{\alpha_{r}} \subseteq \mathfrak{S}_{n}$ be the associated parabolic subgroup of $\mathfrak{S}_{n}$. The factor $\mathfrak{S}_{\alpha_{i}}$ permutes the $i^{t h}$ batch of letters in $[n]$. We also let $L_{\alpha}$ be the Levi subgroup of $G L_{n}(\mathbb{C})$ attached to $\alpha$. Elements of $L_{\alpha}$ are block diagonal matrices of the form $A_{1} \oplus \cdots \oplus A_{r}$ where $A_{i} \in G L_{\alpha_{i}}$. For example, matrices in $L_{(2,1,2)}$ have the form

$$
\left(\begin{array}{lllll}
\star & \star & 0 & 0 & 0 \\
\star & \star & 0 & 0 & 0 \\
0 & 0 & \star & 0 & 0 \\
0 & 0 & 0 & \star & \star \\
0 & 0 & 0 & \star & \star
\end{array}\right)
$$

where the $\star$ 's are complex numbers and three diagonal blocks are invertible.

An ordered set partition of $[n]$ is a sequence $\sigma=\left(B_{1}|\cdots| B_{k}\right)$ of nonempty subsets of $[n]$ such that $[n]=B_{1} \sqcup \cdots \sqcup B_{k}$ (disjoint union). We say that $\sigma$ has size $n$ and $k$ blocks. Let $\mathcal{O P}_{n, k}$ be the family of ordered set partitions of $[n]$ with $k$ blocks. For example, we have $(25|3| 14) \in \mathcal{O P}_{5,3}$.

Suppose $\alpha \in[k]^{r}$ with $|\alpha|=n$. We consider the following collection $\mathcal{O P}_{\alpha, k}$ of ordered set partitions:

$$
\mathcal{O P} \mathcal{P}_{\alpha, k}:=\left\{\begin{array}{c}
\text { for each } 1 \leq i \leq \ell(\alpha), \text { the } i^{t h} \text { batch of letters } \\
\sigma \in \mathcal{O P}_{n, k}: \quad \alpha_{1}+\cdots+\alpha_{i-1}+1, \alpha_{1}+\cdots+\alpha_{i-1}+2, \ldots, \alpha_{1}+\cdots+\alpha_{i} \\
\text { are contained in distinct blocks of } \sigma
\end{array}\right\} .
$$

If $\alpha=(2,1,2)$ and $k=3$ then $(25|3| 14) \in \mathcal{O} \mathcal{P}_{\alpha, k}$ but $(45|2| 13) \notin \mathcal{O P} \mathcal{P}_{\alpha, k}$ (because 4 and 5 lie in the same block).

For any $\alpha \in[k]^{r}$ with $|\alpha|=n$, we have $\mathcal{O P} \mathcal{P}_{\alpha, k} \subseteq \mathcal{O P} \mathcal{P}_{n, k}$. The set $\mathcal{O P}_{n, k}$ carries an action of $\mathfrak{S}_{n}$ by letter permutation. The subset $\mathcal{O} \mathcal{P}_{\alpha, k}$ is typically not closed under the action of the fully symmetric group $\mathfrak{S}_{n}$, but is stable under the action of the parabolic subgroup $\mathfrak{S}_{\alpha}$.

Let $k \geq 0$. A set sequence in $[k]$ is a finite sequence $\mathcal{I}=\left(I_{1}, \ldots, I_{r}\right)$ of nonempty subsets of $[k]$. The type of $\mathcal{I}$ is the composition $\left(\left|I_{1}\right|, \ldots,\left|I_{r}\right|\right) \in[k]^{r}$ obtained by taking the cardinalities of the sets. The sequence $\mathcal{I}$ is said to cover $[k]$ if $I_{1} \cup \cdots \cup I_{r}=[k]$. We omit set braces when writing set sequences, so that when $k=3$ the sequence $(13,3,23,1)$ is a set sequence of type $(2,1,2,1)$ which covers [3] whereas $(23,2,23,3)$ is a set sequence of type $(2,1,2,1)$ which does not cover [3]. We have a natural correspondence

$$
\mathfrak{S}_{\alpha} \text {-orbits in } \mathcal{O P} \mathcal{P}_{\alpha, k} \leftrightarrow \text { set sequences of type } \alpha \text { which cover }[k] \text {. }
$$

We can view set sequences as 0,1-matrices, so that the examples of the last paragraph become

$$
(13,3,23,1) \leftrightarrow\left(\begin{array}{cccc}
1 & 0 & 0 & 1 \\
0 & 0 & 1 & 0 \\
1 & 1 & 1 & 0
\end{array}\right) \quad \text { and } \quad(23,2,23,3) \leftrightarrow\left(\begin{array}{cccc}
0 & 0 & 0 & 0 \\
1 & 1 & 1 & 0 \\
1 & 0 & 1 & 1
\end{array}\right)
$$

The type of the set sequence is the column sum vector of the matrix. Covering set sequences correspond to matrices without zero rows. 
2.2. Gröbner theory. A total order $<$ on the monomials in $\mathbb{Q}\left[\mathbf{x}_{n}\right]$ is a monomial order if

(1) $1 \leq m$ for any monomial $m$ and

(2) if $m_{1}, m_{2}, m_{3}$ are monomials and $m_{1}<m_{2}$, then $m_{1} \cdot m_{3}<m_{2} \cdot m_{3}$.

In this paper, we shall only consider the monomial order defined by $x_{1}^{a_{1}} \cdots x_{n}^{a_{n}}<x_{1}^{b_{1}} \cdots x_{n}^{b_{n}}$ if there exists $1 \leq i \leq n$ such that $a_{i}<b_{i}$ and $a_{i+1}=b_{i+1}, \ldots, a_{n}=b_{n}$. This is the lexicographical order with respect to the reversed variable set $x_{n}>\cdots>x_{1}$. This 'negative lexicographical' order is called neglex.

If $<$ is a monomial order and $f \in \mathbb{Q}\left[\mathbf{x}_{n}\right]$ is a nonzero polynomial, let $\operatorname{in}_{<}(f)$ be the leading monomial of $f$ with respect to $<$. If $I \subseteq \mathbb{Q}\left[\mathbf{x}_{n}\right]$ is an ideal, the initial ideal in $<(I) \subseteq \mathbb{Q}\left[\mathbf{x}_{n}\right]$ is the monomial ideal

$$
\operatorname{in}_{<}(I):=\left\langle\operatorname{in}_{<}(f): f \in I-\{0\}\right\rangle .
$$

Let $I \subseteq \mathbb{Q}\left[\mathbf{x}_{n}\right]$ be an ideal. A finite subset $G=\left\{g_{1}, \ldots, g_{t}\right\} \subseteq I$ is a Gröbner basis of $I$ if $\operatorname{in}_{<}(I)=\left\{\operatorname{in}_{<}\left(g_{1}\right), \ldots\right.$, in $\left._{<}\left(g_{t}\right)\right\}$. Equivalently, the leading term of any nonzero polynomial in $I$ is divisible by the leading term of one of the polynomials in $G$. If $G$ is a Gröbner basis for $I$, we have $I=\langle G\rangle$ as ideals.

2.3. Demazure characters. For $1 \leq i \leq n-1$, the divided difference operator $\partial_{i}: \mathbb{Z}\left[\mathbf{x}_{n}\right] \rightarrow \mathbb{Z}\left[\mathbf{x}_{n}\right]$ is defined by

$$
\partial_{i}\left(f\left(\mathbf{x}_{n}\right)\right):=\frac{f\left(x_{1}, \ldots, x_{i}, x_{i+1}, \ldots, x_{n}\right)-f\left(x_{1}, \ldots, x_{i+1}, x_{i}, \ldots, x_{n}\right)}{x_{i}-x_{i+1}} .
$$

The isobaric divided difference operator $\pi_{i}: \mathbb{Z}\left[\mathbf{x}_{n}\right] \rightarrow \mathbb{Z}\left[\mathbf{x}_{n}\right]$ is given by $\pi_{i}\left(f\left(\mathbf{x}_{n}\right)\right):=\partial_{i}\left(x_{i} f\left(\mathbf{x}_{n}\right)\right)$.

A weak composition $\gamma$ of length $n$ is a sequence $\gamma=\left(\gamma_{1}, \ldots, \gamma_{n}\right)$ of $n$ nonnegative integers. The Demazure character (or key polynomial) $\kappa_{\gamma}\left(\mathbf{x}_{n}\right) \in \mathbb{Z}\left[\mathbf{x}_{n}\right]$ is a polynomial defined recursively by the following two rules.

(1) If $\gamma_{1} \geq \cdots \geq \gamma_{n}$ then $\kappa_{\gamma}\left(\mathbf{x}_{n}\right)$ is the monomial $x_{1}^{\gamma_{1}} \cdots x_{n}^{\gamma_{n}}$.

(2) If $\gamma_{i}<\gamma_{i+1}$ then

$$
\kappa_{\left(\gamma_{1}, \ldots, \gamma_{i+1}, \gamma_{i}, \ldots, \gamma_{n}\right)}\left(\mathbf{x}_{n}\right)=\pi_{i}\left(\kappa_{\left(\gamma_{1}, \ldots, \gamma_{i}, \gamma_{i+1}, \ldots, \gamma_{n}\right)}\left(\mathbf{x}_{n}\right)\right) .
$$

Since the isobaric divided difference operators satisfy the 0 -Hecke relations

$$
\begin{cases}\pi_{i}^{2}=\pi_{i} & \text { for } 1 \leq i \leq n-1, \\ \pi_{i} \pi_{j}=\pi_{j} \pi_{i} & \text { for }|i-j|>1 \\ \pi_{i} \pi_{i+1} \pi_{i}=\pi_{i+1} \pi_{i} \pi_{i+1} & \text { for } 1 \leq i \leq n-2\end{cases}
$$

these rules give a well-defined polynomial $\kappa_{\gamma}\left(\mathbf{x}_{n}\right)$ for any weal composition $\gamma$ of length $n$. We will need the following two properties of $\kappa_{\gamma}\left(\mathbf{x}_{n}\right)$ :

(1) the leading monomial of $\kappa_{\gamma}\left(\mathbf{x}_{n}\right)$ with respect to the neglex order is $x_{1}^{\gamma_{1}} \cdots x_{n}^{\gamma_{n}}$, and

(2) the coefficients of $\kappa_{\gamma}\left(\mathbf{x}_{n}\right)$ are integers and the coefficient of the monomial $x_{1}^{\gamma_{1}} \cdots x_{n}^{\gamma_{n}}$ is 1 .

2.4. Cohomology. We refer the reader to [3] for a thorough reference on the geometric objects used in this paper.

An affine paving of a complex variety $X$ is a sequence

$$
\varnothing=X_{0} \subset X_{1} \subset \cdots \subset X_{m}=X
$$

of closed subvarieties of $X$ such that for $1 \leq i \leq m$, the difference $X_{i}-X_{i-1}$ is isomorphic to a disjoint union $\bigsqcup_{j} A_{i j}$ of complex affine spaces $A_{i j}$. The affine spaces $A_{i j}$ are called the cells of the affine paving and we say that the set $\left\{A_{i j}\right\}$ of all cells induces an affine paving of $X$.

Let $X$ be a smooth irreducible complex algebraic variety. Assume that $X$ admits an affine paving $\varnothing=X_{0} \subset X_{1} \subset \cdots \subset X_{m}=X$. We will need the following two geometric facts. 
(1) The (singular) cohomology $H^{\bullet}(X ; \mathbb{Z})$ is a free $\mathbb{Z}$-module of rank equal to the total number of cells $A_{i j}$ in the affine paving. The classes of the Zariski closures $\left[\overline{A_{i j}}\right]$ of these cells form a $\mathbb{Z}$-basis of $H^{\bullet}(X ; \mathbb{Z})$.

(2) For $1 \leq i \leq m$, the inclusion $\iota: X-X_{i} \hookrightarrow X$ induces a surjective ring homomorphism on cohomology

$$
\iota^{*}: H^{\bullet}(X ; \mathbb{Z}) \rightarrow H^{\bullet}\left(X-X_{i} ; \mathbb{Z}\right) .
$$

Let $X$ be a complex algebraic variety and let $V \rightarrow X$ be a complex vector bundle of rank $r$. For $1 \leq i \leq r$ we have a Chern class $c_{i}(V) \in H^{2 i}(X ; \mathbb{Z})$. Introducing a variable $t$, the Chern polynomial $c_{\bullet}(V)$ is the generating function of these Chern classes:

$$
c_{\bullet}(V):=1+c_{1}(V) t+c_{2}(V) t^{2}+\cdots+c_{r}(V) t^{r} .
$$

Let $p: X^{\prime} \rightarrow X$ be the fiber bundle whose fiber over a point $x \in X$ is the space of complete flags in the $r$-dimensional vector space $V_{x}$. The induced map $p^{*}: H^{\bullet}(X ; \mathbb{Z}) \rightarrow H^{\bullet}\left(X^{\prime} ; \mathbb{Z}\right)$ is injective, so we may view $H^{\bullet}(X ; \mathbb{Z})$ as a subring of $H^{\bullet}\left(X^{\prime} ; \mathbb{Z}\right)$. By the Splitting Principle, there exist (unique up to permutation) elements $x_{1}, \ldots, x_{r} \in H^{2}\left(X^{\prime} ; \mathbb{Z}\right)$ such that $c_{\bullet}(V)=\left(1+x_{1} t\right) \cdots\left(1+x_{r} t\right)$. The degree 2 cohomology elements $x_{1}, \ldots, x_{r}$ are the Chern roots of $V$. We have $c_{i}(V)=e_{i}\left(x_{1}, \ldots, x_{r}\right)$, where $e_{i}$ is the elementary symmetric polynomial of degree $i$. Any symmetric polynomial in $x_{1}, \ldots, x_{r}$ lies in $H^{\bullet}(X ; \mathbb{Z})$.

If $V$ and $W$ are two complex vector bundles over $X$, we can form the direct sum bundle $V \oplus W$. The Whitney sum formula gives the following relation between the Chern polynomials of these bundles:

$$
c_{\bullet}(V \oplus W)=c_{\bullet}(V) \cdot c_{\bullet}(W)
$$

\section{QUOTIENT RINGS}

A subalgebra of the following quotient ring will present the cohomology of $X_{\alpha, k}$. It will be convenient to consider versions of this ring over both $\mathbb{Z}$ and $\mathbb{Q}$.

Definition 3.1. Let $k>0$ and let $\alpha=\left(\alpha_{1}, \ldots, \alpha_{r}\right) \in[k]^{r}$ with $|\alpha|=n$. Let $I_{\alpha, k} \subseteq \mathbb{Z}\left[x_{1}, \ldots, x_{n}\right]$ be the ideal generated by

- the top $k$ elementary symmetric polynomials

$$
e_{n}\left(\mathbf{x}_{n}\right), e_{n-1}\left(\mathbf{x}_{n}\right), \ldots, e_{n-k+1}\left(\mathbf{x}_{n}\right)
$$

in the full variable set $\mathbf{x}_{n}=\left(x_{1}, \ldots, x_{n}\right)$ and

- the complete homogeneous symmetric polynomials

$$
h_{k-\alpha_{i}+1}\left(\mathbf{x}_{n}^{(i)}\right), h_{k-\alpha_{i}+2}\left(\mathbf{x}_{n}^{(i)}\right), \ldots, h_{k}\left(\mathbf{x}_{n}^{(i)}\right) \quad(1 \leq i \leq r)
$$

in the $i^{\text {th }}$ batch of variables $\mathbf{x}_{n}^{(i)}=\left(x_{\alpha_{1}+\cdots+\alpha_{i-1}+1}, \ldots, x_{\alpha_{1}+\cdots+\alpha_{i-1}+\alpha_{i}}\right)$.

Write $R_{\alpha, k}:=\mathbb{Z}\left[\mathbf{x}_{n}\right] / I_{\alpha, k}$ for the corresponding quotient ring.

Let $J_{\alpha, k} \subseteq \mathbb{Q}\left[\mathbf{x}_{n}\right]$ be the ideal in the polynomial ring with rational coefficients having the same generators as $I_{\alpha, k}$. Write $S_{\alpha, k}:=\mathbb{Q}\left[\mathbf{x}_{n}\right] / J_{\alpha, k}$ for the corresponding quotient.

Both of the rings $R_{\alpha, k}$ and $S_{\alpha, k}$ are graded algebras (over $\mathbb{Z}$ and $\mathbb{Q}$, respectively) and carry a graded action of the parabolic subgroup $\mathfrak{S}_{\alpha}$ of $\mathfrak{S}_{n}$. These rings are related by extension of coefficients: $S_{\alpha, k}=\mathbb{Q} \otimes_{\mathbb{Z}} R_{\alpha, k}$.

Although the $\mathbb{Z}$-algebra $R_{\alpha, k}$ will be more useful for cohomology presentation, the $\mathbb{Q}$-algebra $S_{\alpha, k}$ is easier to study directly, and $S_{\alpha, k}$ is the object of study in this section. In Section 5 we will apply Gröbner theory and a Demazure character identity from [7] to bootstrap results to $R_{\alpha, k}$. 
3.1. A slightly unusual $\mathfrak{S}_{n}$-action. The symmetric group $\mathfrak{S}_{n}$ acts on the set $\mathbb{Z}_{\geq 0}^{n}$ of length $n$ words over the nonnegative integers by the rule

$$
\pi .\left(a_{1} \ldots a_{n}\right):=a_{\pi(1)} \ldots a_{\pi(n)}, \quad \pi \in \mathfrak{S}_{n}, a_{1} \ldots a_{n} \in \mathbb{Z}_{\geq 0}^{n} .
$$

In our analysis of the ring $S_{\alpha, k}$, we will use a less standard action of $\mathfrak{S}_{n}$ on $\mathbb{Z}_{\geq 0}^{n}$.

For $1 \leq i \leq n-1$, let $s_{i}=(i, i+1) \in \mathfrak{S}_{n}$ be the corresponding adjacent transposition. For any word $a_{1} \ldots a_{n} \in \mathbb{Z}_{\geq 0}^{n}$, we define a new word $s_{i} \cdot\left(a_{1} \ldots a_{n}\right)$ by the rule

$$
s_{i} \cdot\left(a_{1} \ldots a_{i} a_{i+1} \ldots a_{n}\right):= \begin{cases}a_{1} \ldots a_{i+1}\left(a_{i}-1\right) \ldots a_{n} & \text { if } a_{i}>a_{i+1}, \\ a_{1} \ldots\left(a_{i+1}+1\right) a_{i} \ldots a_{n} & \text { if } a_{i} \leq a_{i+1} .\end{cases}
$$

For example, if $n=4$ and $a_{1} a_{2} a_{3} a_{4}=3225$ then

$$
s_{1} \cdot(3225)=2225, \quad s_{2} \cdot(3225)=3325, \quad s_{3} \cdot(3225)=3242 .
$$

Proposition 3.2. The rule (3.2) extends to give a free action of $\mathfrak{S}_{n}$ on the set of all words in $\mathbb{Z}_{\geq 0}^{n}$. Furthermore, every $\mathfrak{S}_{n}$-orbit in $\mathbb{Z}_{\geq 0}^{n}$ under this action contains a unique strictly decreasing word.

The action $\pi \cdot(-)$ afforded by (3.2) is different from the more traditional action $\pi$.(-). In particular, the action $\pi \cdot(-)$ is free whereas $\pi \cdot(-)$ is not.

Proof. These assertions become evident when we look at the rule (3.2) in a different way. For $n \geq 0$, let $\mathcal{B}_{n}$ be the family of words $b_{1} \ldots b_{m}$ (of any length $m \geq n$ ) over the alphabet $\{0,1,2, \ldots, n\}$ such that $a_{1}>0$, each of the positive letters $1,2, \ldots, n$ appears exactly once, and the letter 0 appears $m-n$ times. We describe a bijection between $\mathcal{B}_{n}$ and $\mathbb{Z}_{>0}^{n}$.

Given a word $b_{1} \ldots b_{m} \in \mathcal{B}_{n}$, the inversion code is the sequence $\left(i_{1}, \ldots, i_{n}\right) \in \mathbb{Z}_{\geq 0}$ where for $1 \leq j \leq n$ the entry $i_{j}$ is given by

$$
i_{j}:=\left|\left\{b_{r+1}, b_{r+2}, \ldots, b_{m}\right\} \cap\{0,1, \ldots, j-1\}\right|, \quad \text { where } a_{r}=j .
$$

The number $i_{j}$ counts inversions in $b_{1} \ldots b_{m}$ starting at $j$. For example, the inversion code of $1402300 \in \mathcal{B}_{4}$ is $(3,2,2,5)$ and (interchanging 3 and 4) the inversion code of $1302400 \in \mathcal{A}_{4}$ is $(3,2,4,2)$.

Since we insist that words in $\mathcal{B}_{n}$ start with positive letters, the association $b_{1} \ldots b_{m} \mapsto\left(i_{1}, \ldots, i_{n}\right)$ of a word to its inversion code gives a bijection $\mathcal{B}_{n} \stackrel{\sim}{\rightarrow} \mathbb{Z}_{\geq 0}^{n}$. The set $\mathcal{B}_{n}$ carries a free action of $\mathfrak{S}_{n}$, where permutations $\pi \in \mathfrak{S}_{n}$ act on positive letters. The formula (3.2) is the transfer of this action to $\mathbb{Z}_{>0}^{n}$ under the inversion code bijection. The unique strictly decreasing word a given $\mathfrak{S}_{n}$-orbit in $\mathbb{Z}_{\geq 0}^{n}$ is the unique word in the corresponding orbit of $\mathcal{B}_{n}$ in which the positive letters appear in decreasing order.

3.2. Orbit harmonics. We want to describe a Gröbner basis for $J_{\alpha, k}$, give the standard monomial basis of $S_{\alpha, k}$, and determine the isomorphism type of $S_{\alpha, k}$ as an ungraded $\mathfrak{S}_{\alpha}$-module. Our basic technique is the method of orbit harmonics; see [1] for a more leisurely exposition.

Let $Z \subseteq \mathbb{Q}^{n}$ be a finite set of points. We associate to $Z$ the ideal $\mathbf{I}(Z) \subseteq \mathbb{Q}\left[\mathbf{x}_{n}\right]$ of polynomials which vanish on $Z$ :

$$
\mathbf{I}(Z):=\left\{f\left(\mathbf{x}_{n}\right) \in \mathbb{Q}\left[\mathbf{x}_{n}\right]: f\left(\mathbf{z}_{n}\right)=0 \text { for all } \mathbf{z}_{n} \in Z\right\} .
$$

By Lagrange Interpolation, we can identify the quotient $\mathbb{Q}\left[\mathbf{x}_{n}\right] / \mathbf{I}(Z)$ with the $\mathbb{Q}$-vector space $\mathbb{Q}[Z]$ of all functions $Z \rightarrow \mathbb{Q}$. In particular, we have $\operatorname{dim}\left(\mathbb{Q}\left[x_{1}, \ldots, x_{n}\right] / \mathbf{I}(Z)\right)=|Z|$.

The ideal $\mathbf{I}(Z)$ is typically inhomogeneous. To get a homogeneous ideal we proceed as follows. For any nonzero polynomial $f \in \mathbb{Q}\left[\mathbf{x}_{n}\right]$ let $f=f_{d}+\cdots+f_{1}+f_{0}$ where $f_{i}$ is homogeneous of degree $i$ and $f_{d} \neq 0$. Write $\tau(f):=f_{d}$ for the top degree component of $f$. Define an ideal $\mathbf{T}(Z) \subseteq \mathbb{Q}\left[x_{1}, \ldots, x_{n}\right]$ by the rule

$$
\mathbf{T}(Z):=\langle\tau(f): f \in \mathbf{I}(Z), f \neq 0\rangle
$$


The ideal $\mathbf{T}(Z)$ is homogeneous by construction, so that $\mathbb{Q}\left[\mathbf{x}_{n}\right] / \mathbf{T}(Z)$ is a graded $\mathbb{Q}$-algebra. We have isomorphisms of ungraded $\mathbb{Q}$-vector spaces

$$
\mathbb{Q}\left[\mathbf{x}_{n}\right] / \mathbf{T}(Z) \cong \mathbb{Q}\left[x_{1}, \ldots, x_{n}\right] / \mathbf{I}(Z) \cong \mathbb{Q}[Z] .
$$

Let $G \subseteq G L_{n}(\mathbb{Q})$ be any finite matrix group acting on $\mathbb{Q}^{n}$ canonically and on $\mathbb{Q}\left[\mathbf{x}_{n}\right]$ by linear substitution. When the point set $Z \subseteq \mathbb{Q}^{n}$ is stable under the action of $G$, the ideals $\mathbf{I}(Z)$ and $\mathbf{T}(Z)$ are also $G$-stable and (3.6) is a sequence of ungraded $G$-module isomorphisms. In our setting, the group $G$ will be the parabolic subgroup $\mathfrak{S}_{\alpha}$ of $\mathfrak{S}_{n}$, viewed as a group of permutation matrices. Our point locus $Z$ is given as follows.

Definition 3.3. Let $\alpha \in[k]^{r}$ be a composition with $|\alpha|=n$. Fix $k$ distinct rational numbers $\eta_{1}, \ldots, \eta_{k}$. Let $Z_{\alpha, k} \subseteq \mathbb{Q}^{n}$ be the set of points $\left(z_{1}, \ldots, z_{n}\right)$ such that

- $\left\{z_{1}, \ldots, z_{n}\right\}=\left\{\eta_{1}, \ldots, \eta_{k}\right\}$ and

- for $1 \leq i \leq r$ the $i^{t h}$ batch of coordinates $z_{\alpha_{1}+\cdots+\alpha_{i-1}+1}, \ldots, z_{\alpha_{1}+\cdots+\alpha_{i-1}+\alpha_{i}}$ are distinct.

The point set $Z_{\alpha, k}$ is stable under the action of $\mathfrak{S}_{\alpha}$. There is a natural correspondence between $\mathcal{O P} \mathcal{P}_{\alpha, k}$ and $Z_{\alpha, k} ;$ in the case $\alpha=(2,1,2), k=3$ we have

$$
(23|4| 15) \leftrightarrow\left(\eta_{3}, \eta_{1}, \eta_{1}, \eta_{2}, \eta_{3}\right)
$$

Under this correspondence we have the identification of ungraded $\mathfrak{S}_{\alpha}$-modules

$$
\mathbb{Q}\left[\mathcal{O P} \mathcal{L}_{\alpha, k}\right]=\mathbb{Q}\left[Z_{\alpha, k}\right]
$$

We get a lower bound for the dimension of $S_{\alpha, k}$.

Lemma 3.4. Let $\alpha \in[k]^{r}$ satisfy $|\alpha|=n$. We have $J_{\alpha, k} \subseteq \mathbf{T}\left(Z_{\alpha, k}\right)$.

We will prove the equality $J_{\alpha, k}=\mathbf{T}\left(Z_{\alpha, k}\right)$ in the next subsection.

Proof. We show that each generator of $J_{\alpha, k}$ lies in $\mathbf{T}\left(Z_{\alpha, k}\right)$. We begin with the generators of the form $e_{d}\left(x_{1}, \ldots, x_{n}\right)$ for $n-k+1 \leq d \leq n$. Introduce a new variable $t$ and consider the rational function

$$
\frac{\left(1+x_{1} t\right)\left(1+x_{2} t\right) \cdots\left(1+x_{n} t\right)}{\left(1+\eta_{1} t\right)\left(1+\eta_{2} t\right) \cdots\left(1+\eta_{k} t\right)}
$$

When $\left(x_{1}, \ldots, x_{n}\right) \in Z_{\alpha, k}$, the $k$ factors in the denominator cancel with $k$ factors in the numerator to give a polynomial in $t$ of degree $n-k$. For any $n-k+1 \leq d \leq n$, the coefficient of $t^{d}$ in this expression is then

$$
0=\sum_{j=0}^{d}(-1)^{j} e_{d-j}\left(\mathbf{x}_{n}\right) h_{j}\left(\eta_{1}, \ldots, \eta_{k}\right) .
$$

Taking the top degree component, we see that $e_{d}\left(\mathbf{x}_{n}\right) \in \mathbf{T}\left(Z_{\alpha, k}\right)$.

Now fix $1 \leq i \leq r$ and consider the rational function

$$
\frac{\left(1-\eta_{1} t\right)\left(1-\eta_{2} t\right) \cdots\left(1-\eta_{k} t\right)}{\left(1-x_{\alpha_{1}+\cdots+\alpha_{i-1}+1} t\right) \cdots\left(1+x_{\alpha_{1}+\cdots+\alpha_{i-1}+\alpha_{i}} t\right)}
$$

involving the $i^{\text {th }}$ batch of variables $\mathbf{x}_{n}^{(i)}$. When $\left(x_{1}, \ldots, x_{n}\right) \in Z_{\alpha, k}$, the $\alpha_{i}$ factors in the denominator cancel with $\alpha_{i}$ factors in the numerator to give a polynomial in $t$ of degree $k-\alpha_{i}$. In particular, for any $d>k-\alpha_{i}$, the coefficient of $t^{d}$ in this polynomial is

$$
0=\sum_{j=0}^{k}(-1)^{j} h_{d-j}\left(\mathbf{x}_{n}^{(i)}\right) e_{j}\left(\eta_{1}, \ldots, \eta_{k}\right) .
$$

Taking the top degree component, we get $h_{d}\left(\mathbf{x}_{n}^{(i)}\right) \in \mathbf{T}\left(Z_{\alpha, k}\right)$. 
Lemma 3.4 tells us that $\operatorname{dim}\left(S_{\alpha, k}\right) \geq\left|\mathcal{O} \mathcal{P}_{\alpha, k}\right|$. To get the reverse inequality, we will need the unusual action of $\mathfrak{S}_{n}$ (or rather its parabolic subgroup $\mathfrak{S}_{\alpha}$ ) afforded by Proposition 3.2 .

3.3. The structure of $S_{\alpha, k}$. For any subset $S=\left\{s_{1}<s_{2}<\cdots<s_{t}\right\} \subseteq[n]$, the skip sequence $\gamma(S)=\left(\gamma_{1}, \ldots, \gamma_{n}\right)$ is defined by

$$
\gamma_{i}= \begin{cases}i-j+1 & \text { if } i=s_{j} \in S, \\ 0 & \text { if } i \notin S .\end{cases}
$$

The reverse skip sequence is $\gamma(S)^{*}:=\left(\gamma_{n}, \ldots, \gamma_{1}\right)$. For example, if $n=8$ and $S=\{2,3,5,8\}$ then $\gamma(S)=(0,2,2,0,3,0,0,5)$ and $\gamma(S)^{*}=(5,0,0,3,0,2,2,0)$. We also let $\mathbf{x}(S)^{*}:=x_{1}^{\gamma_{n}} \cdots x_{n}^{\gamma_{1}}$ be the reverse skip monomial, so that $\mathbf{x}(S)^{*}=x_{1}^{5} x_{4}^{3} x_{6}^{2} x_{7}^{2}$ in our example. The terminology comes from the fact that nonzero entries of skip sequences $\gamma(S)$ increment whenever the set $S$ skips an element.

Definition 3.5. Let $\alpha \in[k]^{r}$ satisfy $|\alpha|=n$ and let $m \in \mathbb{Q}\left[x_{1}, \ldots, x_{n}\right]$ be a monomial. The monomial $m$ is $\alpha$-nonskip if

- for any subset $S \subseteq[n]$ with $|S|=n-k+1$ we have $\mathbf{x}(S)^{*} \nmid m$, and

- for $1 \leq i \leq r$ and $1 \leq j \leq \alpha_{i}$, the $j^{\text {th }}$ variable in the $i^{\text {th }}$ batch of variables satisfies $x_{\alpha_{1}+\cdots+\alpha_{i-1}+j}^{k-j+1} \nmid m$.

Let $\mathcal{M}_{\alpha, k}$ be the set of $\alpha$-nonskip monomials in $\mathbb{Q}\left[x_{1}, \ldots, x_{n}\right]$.

The set $\mathcal{M}_{\alpha, k}$ will turn out to be the standard monomial basis for $S_{\alpha, k}$ under the neglex term order. To show that $\mathcal{M}_{\alpha, k}$ contains this standard monomial basis, we quote the following result of Haglund, the author, and Shimozono [7. This polynomial identity will be crucial for our work.

Theorem 3.6. (Haglund-R.-Shimozono [7, Lem. 3.4]) Let $S \subseteq[n]$ satisfy $|S|=n-k+1$. We have the following identity involving Demazure characters:

$$
\kappa_{\gamma(S)^{*}}\left(\mathbf{x}_{n}\right)=\sum_{\lambda}(-1)^{|\lambda|} \kappa \overline{\gamma(S)^{*}-\lambda}\left(\mathbf{x}_{n}\right) e_{n-k+1+|\lambda|}\left(\mathbf{x}_{n}\right) .
$$

Here $\gamma(S)^{*}=\left(\gamma_{n}, \ldots, \gamma_{1}\right)$ is the reverse skip sequence of $S$, the sequence $\overline{\gamma(S)^{*}}$ is the componentwise maximum of the length $n$ sequences $\left(\gamma_{n}-1, \ldots, \gamma_{1}-1\right)$ and the length $n$ sequence of zeroes $(0, \ldots, 0)$, and the sum is over subsets $\lambda$ of the skyline diagram of $\gamma(S)^{*}$ which are left leaning.

We will not need the notion of a skyline diagram, or a left leaning subset thereof, in this paper. It suffices for us to notice that

if $\alpha \in[k]^{r}$ with $|\alpha|=n$, the Demazure character $\kappa_{\gamma(S)^{*}}\left(\mathbf{x}_{n}\right)$ corresponding to the reverse skip sequence $\gamma(S)^{*}$ may be expressed as a $\mathbb{Z}\left[\mathbf{x}_{n}\right]$-linear combination of $e_{n}\left(\mathbf{x}_{n}\right), e_{n-1}\left(\mathbf{x}_{n}\right), \ldots, e_{n-k+1}\left(\mathbf{x}_{n}\right)$.

It is this central fact that will allow us to

- describe the Gröbner basis and ungraded $\mathfrak{S}_{\alpha}$-isomorphism type of $S_{\alpha, k}$ and

- present the integral (rather than just rational) cohomology $H^{\bullet}\left(X_{\alpha, k} ; \mathbb{Z}\right)$ as the $\mathfrak{S}_{\alpha}$-invariant subring $\left(R_{\alpha, k}\right)^{\mathfrak{S}_{\alpha}}$ of $R_{\alpha, k}$.

Theorem 3.6 will help us go back and forth between $\mathbb{Q}$-vector spaces and $\mathbb{Z}$-modules.

The proof of Theorem [3.6 in [7] uses a sign-reversing involution and an extension of the dual Pieri rule to Demazure characters appearing in a 2011 paper of Hagund, Luoto, Mason, and van Willigenburg [5]. Given the elegant definition of the spaces $X_{\alpha, k}$ of spanning configurations, it is natural to wonder whether their integral cohomology could be presented using pre-2011 technology.

Here is our first application of Theorem 3.6.

Lemma 3.7. Let $\alpha \in[k]^{r}$ with $|\alpha|=n$. The following monomials are contained in the initial ideal in $_{<}\left(J_{\alpha, k}\right)$ under the neglex term order: 
- the reverse skip monomial $\mathbf{x}(S)^{*}$, where $S \subseteq[n]$ satisfies $|S|=n-k+1$ and

- the variable power $x_{\alpha_{1}+\cdots+\alpha_{i-1}+j}^{k-j+1}$ involving the $j^{\text {th }}$ variable from the $i^{\text {th }}$ batch of variables, where $1 \leq i \leq r$ and $1 \leq j \leq \alpha_{i}$.

Consequently, the set $\mathcal{M}_{\alpha, k}$ of $\alpha$-nonskip monomials contains the standard monomial basis of $S_{\alpha, k}$.

Proof. Let $S \subseteq[n]$ satisfy $|S|=n-k+1$. By Theorem 3.6, the Demazure character $\kappa_{\gamma(S)^{*}}\left(x_{1}, \ldots, x_{n}\right)$ lies in the ideal $J_{\alpha, k}$. Since the neglex-leading term of $\kappa_{\gamma(S)^{*}}\left(x_{1}, \ldots, x_{n}\right)$ is $\mathbf{x}(S)^{*}$, we have the first bullet point. For the second bullet point, the identity

$$
h_{d}\left(y_{1}, \ldots, y_{m}\right)=h_{d}\left(y_{1}, \ldots, y_{m-1}\right)+y_{m} \cdot h_{d-1}\left(y_{1}, \ldots, y_{m}\right), \quad m, d>0
$$

may be used to deduce that

$$
h_{k-j+1}\left(x_{\alpha_{1}+\cdots+\alpha_{i-1}+1}, \ldots, x_{\alpha_{1}+\cdots+\alpha_{i-1}+j}\right) \in J_{\alpha, k}
$$

for each $1 \leq j \leq \alpha_{i}$. Taking the neglex-leading term, we see that $x_{\alpha_{1}+\cdots+\alpha_{i-1}+j}^{k-j+1} \in \operatorname{in}_{<}\left(J_{\alpha, k}\right)$.

Our next task is to prove that $\left|\mathcal{M}_{\alpha, k}\right|=\left|\mathcal{O P}_{\alpha, k}\right|$ for any $\alpha \in[k]^{r}$. There are two main ingredients to our argument: a notion of coinversion code due to the author and Wilson [10] and the unusual $\mathfrak{S}_{n}$-action of Proposition 3.2. We first prove that the restriction of this action to $\mathfrak{S}_{\alpha}$ preserves (the exponent sequences of) monomials in $\mathcal{M}_{\alpha, k}$.

Lemma 3.8. Let $\alpha \in[k]^{r}$ with $|\alpha|=n$. Identify monomials $x_{1}^{a_{1}} \cdots x_{n}^{a_{n}}$ in $\mathbb{Q}\left[\mathbf{x}_{n}\right]$ with their exponent sequences $\left(a_{1}, \ldots, a_{n}\right)$. The set of monomials $\mathcal{M}_{\alpha, k}$ is stable under the action of the parabolic subgroup $\mathfrak{S}_{\alpha} \subseteq \mathfrak{S}_{n}$ given in Proposition 3.2.

Proof. Consider an index $p \in[n-1]-\left\{\alpha_{1}, \alpha_{1}+\alpha_{2}, \ldots\right\}$ corresponding to a typical adjacent transposition $s_{p}=(p, p+1)$ generating $\mathfrak{S}_{\alpha}$. Suppose $m=x_{1}^{a_{1}} \cdots x_{n}^{a_{n}} \in \mathcal{M}_{\alpha, k}$ is $\alpha$-nonskip. We show $s_{p} \cdot m \in \mathcal{M}_{\alpha, k}$ also $\alpha$-nonskip. The application of $s_{p} \cdot(-)$ sends $\left(a_{p}, a_{p+1}\right)=\left(a_{p}^{\prime}, a_{p+1}^{\prime}\right)$ where

$$
\begin{cases}\left(a_{p}^{\prime}, a_{p+1}^{\prime}\right)=\left(a_{p+1}, a_{p}-1\right) & \text { if } a_{p}>a_{p+1}, \\ \left(a_{p}^{\prime}, a_{p+1}^{\prime}\right)=\left(a_{p+1}+1, a_{p}\right) & \text { if } a_{p} \leq a_{p+1}\end{cases}
$$

while leaving the remaining entries of $\left(a_{1}, \ldots, a_{n}\right)$ unchanged.

Suppose $x_{p}$ lies in the $i^{\text {th }}$ batch of variables, so that $x_{p}=x_{\alpha_{1}+\cdots+\alpha_{i-1}+j}$ for some $1 \leq j<\alpha_{i}$. Since $m \in \mathcal{M}_{\alpha, k}$, we have $a_{p}<k-j+1$ and $a_{p+1}<k-j$. Checking both possibilities $a_{p}>a_{p+1}$ and $a_{p} \leq a_{p+1}$, we see that $a_{p}^{\prime}<k-p+1$ and $a_{p+1}^{\prime}<k-p$. This proves that the monomial $s_{j} \cdot m$ satisfies the second bullet point of Definition 3.5.

For the first bullet point of Definition [3.5, write $s_{p} \cdot\left(a_{1}, \ldots, a_{n}\right)=\left(a_{1}^{\prime}, \ldots, a_{n}^{\prime}\right)$ and suppose we have the componentwise inequality $\gamma\left(S^{\prime}\right)^{*} \leq\left(a_{1}^{\prime}, \ldots, a_{n}^{\prime}\right)$ for some subset $S^{\prime} \subseteq[n]$ with $\left|S^{\prime}\right|=$ $n-k+1$. Define a new subset $S \subseteq[n]$ by

$$
S:= \begin{cases}\left(S^{\prime}-\{p\}\right) \cup\{p+1\} & \text { if } p \in S^{\prime} \text { and } p+1 \notin S^{\prime} \\ \left(S^{\prime}-\{p+1\}\right) \cup\{p\} & \text { if } p+1 \in S^{\prime} \text { and } p \notin S^{\prime} \\ S^{\prime} & \text { otherwise. }\end{cases}
$$

One checks the componentwise inequality $\gamma(S) \leq\left(a_{1}, \ldots, a_{n}\right)$, which contradicts the assumption that $m$ is $\alpha$-nonskip. We conclude that $s_{j} \cdot m$ is $\alpha$-nonskip, as desired.

We are ready to show the equality $\left|\mathcal{M}_{\alpha, k}\right|=\left|\mathcal{O P}_{\alpha, k}\right|$. Lemma 3.8 is a key tool in this argument.

Lemma 3.9. Let $\alpha \in[k]^{r}$ with $|\alpha|=n$. We have $\left|\mathcal{M}_{\alpha, k}\right|=\left|\mathcal{O P}_{\alpha, k}\right|$. 
Proof. Let $\sigma=\left(B_{1}|\cdots| B_{k}\right) \in \mathcal{O P}_{n, k}$ be an ordered set partition of $[n]$ with $k$ blocks. The coinversion code code $(\sigma)=\left(c_{1}, \ldots, c_{n}\right)$ of $\sigma$ is the length $n$ sequence whose $i^{\text {th }}$ term is

$$
c_{i}= \begin{cases}\left|\left\{j^{\prime}>j: \min \left(B_{j^{\prime}}\right)>i\right\}\right| & \text { if } i=\min \left(B_{j}\right) \\ \left|\left\{j^{\prime}>j: \min \left(B_{j^{\prime}}\right)>i\right\}\right|+(j-1) & \text { if } i \in B_{j} \text { and } i>\min \left(B_{j}\right) .\end{cases}
$$

The author and Wilson prove [10, Thm. 2.2] that the map $\sigma \mapsto \operatorname{code}(\sigma)$ is a bijection from $\mathcal{O P}_{n, k}$ to sequences $\left(c_{1}, \ldots, c_{n}\right) \in[k]^{n}$ such that for any $S \subseteq[n]$ with $|S|=n-k+1$, the componentwise inequality $\gamma(S)^{*} \leq\left(c_{1}, \ldots, c_{n}\right)$ does not hold.

Identifying monomials with their exponent vectors, if the map $\sigma \mapsto \operatorname{code}(\sigma)$ restricted to give a bijection from $\mathcal{O P} \mathcal{P}_{\alpha, k}$ to $\mathcal{M}_{\alpha, k}$, this would prove the result. Unfortunately, this is not the case. For example, when $\alpha=(2,2)$ and $k=3$ we have $\operatorname{code}(34|1| 2)=(1,0,0,0)$. Since 3 and 4 lie in the same block, $(34|2| 1) \notin \mathcal{O P}_{(2,2)}$ but $x_{1}^{1} x_{2}^{0} x_{3}^{0} x_{4}^{0} \in \mathcal{M}_{\alpha, k}$.

We combine coinversion codes and the action of $\mathfrak{S}_{\alpha}$ on $\mathcal{M}_{\alpha, k}$ coming from Lemma 3.8 to prove the result. A length $n$ sequence $\left(c_{1}, \ldots, c_{n}\right)$ is $\alpha$-decreasing if for $1 \leq i \leq r$ the $i^{\text {th }}$ batch of terms is strictly decreasing:

$$
c_{\alpha_{1}+\cdots+\alpha_{i-1}+1}>c_{\alpha_{1}+\cdots+\alpha_{i-1}+2}>\cdots>c_{\alpha_{1}+\cdots+\alpha_{i-1}+\alpha_{i}}
$$

For example, the sequence $(5,3,0,0,1)$ is $(3,1,1)$-decreasing but not $(1,3,1)$-decreasing or $(1,1,3)$ decreasing. We call a monomial $x_{1}^{c_{1}} \cdots x_{n}^{c_{n}} \alpha$-decreasing if $\left(c_{1}, \ldots c_{n}\right)$ is $\alpha$-decreasing. Let $\overline{\mathcal{M}_{\alpha, k}} \subseteq$ $\mathcal{M}_{\alpha, k}$ be the set of $\alpha$-decreasing $\alpha$-nonskip monomials.

Claim: The coinversion code bijection $\sigma \mapsto \operatorname{code}(\sigma)$ puts $\overline{\mathcal{M}_{\alpha, k}}$ in a one-to-one correspondence with a subset $\overline{\mathcal{O P}} \mathcal{P}_{\alpha, k}$ of $\mathcal{O P} \mathcal{P}_{\alpha, k}$. Furthermore, every element of $\mathcal{O P}{ }_{\alpha, k}$ is $\mathfrak{S}_{\alpha}$-conjugate to a unique element of $\overline{\mathcal{O P}} \mathcal{P}_{\alpha, k}$.

Let us explain how the lemma follows from the claim. The action of $\mathfrak{S}_{\alpha}$ on $\mathcal{O} \mathcal{P}_{\alpha, k}$ is free by the definition of $\mathcal{O P}_{\alpha, k}$. The action of $\mathfrak{S}_{\alpha}$ on $\mathcal{M}_{\alpha, k}$ coming from Lemma 3.8 is free by Proposition 3.2. Also by Proposition 3.2 , every monomial in $\mathcal{M}_{\alpha, k}$ is $\mathfrak{S}_{\alpha}$-conjugate to a unique monomial in $\overline{\mathcal{M}_{\alpha, k}}$. Given the claim, we can use these free actions and the bijection $\overline{\mathcal{M}_{\alpha, k}} \leftrightarrow \overline{\mathcal{O P}_{\alpha, k}}$ to uniquely construct a $\mathfrak{S}_{\alpha}$-equivariant bijection between $\mathcal{M}_{\alpha, k} \leftrightarrow \mathcal{O P}_{\alpha, k}$.

Now we prove the claim. We need to understand the inverse of the coinversion word bijection $\sigma \mapsto \operatorname{code}(\sigma)$. The inverse is constructed recursively by an insertion algorithm; following the notation of [10, Proof of Thm. 2.2], we call this inverse map $\iota$.

Let $\left(B_{1}|\cdots| B_{k}\right)$ be a sequence of of $k$ possibly empty sets of positive integers. We define the coinversion label of the sets $B_{1}, \ldots, B_{k}$ by labeling the empty sets with $0,1, \ldots, j$ from right to left (where there are $j+1$ empty sets), and then labeling the nonempty sets with $j+1, j+2, \ldots, k-1$ from left to right. An example of coinversion labels is as follows, displayed as superscripts:

$$
\left(\varnothing^{2}\left|13^{3}\right| \varnothing^{1}\left|25^{4}\right| 4^{5} \mid \varnothing^{0}\right) .
$$

By construction, each of the letters $0,1, \ldots, k-1$ appears exactly once as a coinversion label.

Let $\left(c_{1}, \ldots, c_{n}\right) \in[k]^{n}$ satisfy $\gamma(S)^{*} \not \leq\left(c_{1}, \ldots, c_{n}\right)$ for all $S \subseteq[n]$ with $|S|=n-k+1$. We define $\iota\left(c_{1}, \ldots, c_{n}\right)=\left(B_{1}|\cdots| B_{k}\right)$ recursively by starting with the sequence $(\varnothing|\cdots| \varnothing)$ of $k$ copies of the empty set, and for $i=1,2, \ldots, n$ inserting $i$ into the unique block with coinversion label $c_{i}$.

Let $n=9, k=4$, and $\left(c_{1}, \ldots, c_{9}\right)=(2,0,3,1,0,0,2,1,0)$. Observe that $\left(c_{1}, \ldots, c_{9}\right)$ is $\alpha$ decreasing where $\alpha=(2,3,1,3)$. We calculate $\iota\left(c_{1}, \ldots, c_{9}\right)$ with the following table. 


\begin{tabular}{c|c|c}
$i$ & $c_{i}$ & $\sigma$ \\
\hline 1 & 2 & $\left(\varnothing^{3}\left|\varnothing^{2}\right| \varnothing^{1} \mid \varnothing^{0}\right)$ \\
2 & 0 & $\left(\varnothing^{2}\left|1^{3}\right| \varnothing^{1} \mid \varnothing^{0}\right)$ \\
3 & 3 & $\left(\varnothing^{1}\left|1^{2}\right| \varnothing^{0} \mid 2^{3}\right)$ \\
4 & 1 & $\left(\varnothing^{1}\left|1^{2}\right| \varnothing^{0} \mid 23^{3}\right)$ \\
5 & 0 & $\left(4^{1}\left|1^{2}\right| \varnothing^{0} \mid 23^{3}\right)$ \\
6 & 0 & $\left(4^{0}\left|1^{1}\right| 5^{2} \mid 23^{3}\right)$ \\
7 & 2 & $\left(46^{0}\left|1^{1}\right| 5^{2} \mid 23^{3}\right)$ \\
8 & 1 & $\left(46^{0}\left|1^{1}\right| 57^{2} \mid 23^{3}\right)$ \\
9 & 0 & $\left(46^{0}\left|18^{1}\right| 57^{2} \mid 23^{3}\right)$
\end{tabular}

We conclude $\iota(2,0,3,1,0,0,2,1,0)=(469|18| 57 \mid 23)$. It is proven in [10, Proof of Thm. 2.2] that $\iota\left(c_{1}, \ldots, c_{n}\right)$ is always an ordered set partition (i.e. that there are no empty blocks at the end of this procedure). Observe that the output $\iota\left(c_{1}, \ldots, c_{n}\right)$ lies in $\mathcal{O P}_{\alpha, k}$.

Assume that the sequence $\left(c_{1}, \ldots, c_{n}\right)$ is both $\alpha$-decreasing and $\alpha$-nonskip. When the algorithm defining $\iota$ processes the $i^{\text {th }}$ batch of letters in $\left(c_{1}, \ldots, c_{n}\right)$ (for some $1 \leq i \leq r$ ), the $\alpha$-decreasing condition makes the algorithm place the $i^{\text {th }}$ batch of numbers in $[n]$ in different blocks (see the above example); this shows that $\iota\left(c_{1}, \ldots, c_{n}\right) \in \mathcal{O P}_{\alpha, k}$. We take $\overline{\mathcal{O P} \mathcal{P}_{\alpha, k}}$ to be the image of $\overline{\mathcal{M}_{\alpha, k}}$ under $\iota$.

It remains to check that every ordered set partition in $\mathcal{O P} \mathcal{P}_{\alpha, k}$ is $\mathfrak{S}_{\alpha}$-conjugate to a unique element in $\overline{\mathcal{O P}} \alpha, k$.

What do elements of $\overline{\mathcal{O P}} \mathcal{P}_{\alpha, k}$ look like? For any element $\sigma=\left(B_{1}|\cdots| B_{k}\right) \in \mathcal{O P}_{\alpha, k}$, consider the sequence $\sigma^{(0)}, \sigma^{(1)}, \ldots, \sigma^{(r)}$ of lists $\sigma^{(i)}=\left(B_{1}^{(i)}|\cdots| B_{k}^{(i)}\right)$ of possibly empty sets of positive integers given by

$$
B_{j}^{(i)}:=B_{j} \cap\left\{1,2, \ldots, \alpha_{1}+\cdots+\alpha_{i}\right\} .
$$

In particular, we have $\sigma^{(0)}=(\varnothing|\cdots| \varnothing)$ and $\sigma^{(r)}=\sigma$. The sequence $\sigma^{(i)}$ is obtained from $\sigma^{(i-1)}$ by adding the $i^{\text {th }}$ batch of letters in $[n]$. The coinversion labels give a total order on the blocks of $\sigma^{(i-1)}$. The partition $\sigma$ is $\alpha, i$-compatible (for $1 \leq i \leq r$ ) if the transformation $\sigma^{(i-1)} \leadsto \sigma^{(i)}$ adds these letters (from smallest to largest) into blocks of strictly decreasing coinversion label with respect to $\sigma^{(i-1)}$. The partition $\sigma$ is $\alpha$-compatible if it is $\alpha, i$-compatible for each $1 \leq i \leq r$.

To see how these concepts work, let $\alpha=(2,3,1,3)$ (so that $r=4$ ) and consider our example ordered set partition $\sigma=(469|18| 57 \mid 23) \in \mathcal{O P}_{9,4}$. The sequences $\sigma^{(0)}, \sigma^{(1)}, \ldots, \sigma^{(4)}$ (together with the relevant coinversion labels) are

$$
\begin{aligned}
\sigma^{(0)} & =\left(\varnothing^{3}\left|\varnothing^{2}\right| \varnothing^{1} \mid \varnothing^{0}\right) \\
\sigma^{(1)} & =\left(\varnothing^{1}\left|1^{2}\right| \varnothing^{0} \mid 2^{3}\right) \\
\sigma^{(2)} & =\left(4^{0}\left|1^{1}\right| 5^{2} \mid 23^{3}\right) \\
\sigma^{(3)} & =\left(46^{0}\left|1^{1}\right| 5^{2} \mid 23^{3}\right) \\
\sigma^{(4)} & =(469|18| 57 \mid 23)
\end{aligned}
$$

We see that the newly added elements at each stage are added in strictly decreasing order with respect to coinversion label, so that $\sigma$ is $\alpha$-compatible. From the definition of $\iota$ we see that $\overline{\mathcal{O P}} \mathcal{P}_{\alpha, k}=\iota\left(\overline{\mathcal{M}_{\alpha, k}}\right)$ is precisely the set of $\alpha$-compatible elements in $\mathcal{O P} \mathcal{P}_{\alpha, k}$.

Each partition $\sigma \in \mathcal{O P}_{\alpha, k}$ is $\mathfrak{S}_{\alpha}$-conjugate to a unique $\alpha$-compatible partition. Therefore, each partition $\sigma \in \mathcal{O} \mathcal{P}_{\alpha, k}$ is $\mathfrak{S}_{\alpha}$-conjugate to a unique element of $\overline{\mathcal{O} \mathcal{P}_{\alpha, k}}$. This completes the proof of the claim.

We are ready to describe the standard monomial basis and $\mathfrak{S}_{\alpha}$-module structure of $S_{\alpha, k}$. 
Theorem 3.10. Let $\alpha \in[k]^{r}$ with $|\alpha|=n$. Endow monomials in $\mathbb{Q}\left[\mathbf{x}_{n}\right]$ with the neglex term order. The set $\mathcal{M}_{\alpha, k}$ of $\alpha$-nonskip monomials is the standard monomial basis of $S_{\alpha, k}$. We have an isomorphism of ungraded $\mathfrak{S}_{\alpha}$-modules

$$
S_{\alpha, k} \cong_{\mathfrak{S}_{\alpha}} \mathbb{Q}\left[\mathcal{O P}{ }_{\alpha, k}\right]
$$

Proof. We have the chain of inequalities

$$
\operatorname{dim}\left(S_{\alpha, k}\right) \leq\left|\mathcal{M}_{\alpha, k}\right|=\left|\mathcal{O P}_{\alpha, k}\right| \leq \operatorname{dim}\left(S_{\alpha, k}\right) .
$$

The first inequality is Lemma 3.7, the equality is Lemma 3.9, and the second inequality is Lemma 3.4 . We conclude that $\operatorname{dim}\left(S_{\alpha, k}\right)=\left|\mathcal{M}_{\alpha, k}\right|=\left|\mathcal{O} \mathcal{P}_{\alpha, k}\right|$.

Lemma 3.7 implies that $\mathcal{M}_{\alpha, k}$ is the standard monomial basis of $S_{\alpha, k}$. Lemma 3.4 and the fact that $\mathbb{Q}\left[x_{1}, \ldots, x_{n}\right] / \mathbf{T}\left(Z_{\alpha, k}\right) \cong \mathbb{Q}\left[\mathcal{O P} \mathcal{P}_{\alpha, k}\right]$ as ungraded $\mathfrak{S}_{\alpha}$-modules imply that $J_{\alpha, k}=\mathbf{T}\left(Z_{\alpha, k}\right)$, which yields the desired $\mathfrak{S}_{\alpha}$-module isomorphism.

We can also write down a Gröbner basis for $J_{\alpha, k}$. We state this result together with a fact about integrality we will need for cohomology computation.

Proposition 3.11. Let $\alpha \in[k]^{r}$ with $|\alpha|=n$. Endow monomials in $\mathbb{Q}\left[\mathbf{x}_{n}\right]$ with the neglex term order. A Gröbner basis for $J_{\alpha, k}$ consists of the Demazure characters

$$
\kappa_{\gamma(S)^{*}}\left(\mathbf{x}_{n}\right), \quad S \subseteq[n],|S|=n-k+1
$$

together with the complete homogeneous symmetric polynomials

$$
h_{k-j+1}\left(x_{\alpha_{1}+\cdots+\alpha_{i-1}+1}, \ldots, x_{\alpha_{1}+\cdots+\alpha_{i-1}+j}\right), \quad 1 \leq i \leq r, 1 \leq j \leq \alpha_{i} .
$$

Every element in the Gröbner basis above is a polynomial in $\mathbb{Z}\left[\mathbf{x}_{n}\right]$ with integer coefficients and neglex-leading coefficient 1. Furthermore, every polynomial in the Gröbner basis above lies in the ideal $I_{\alpha, k} \subseteq \mathbb{Z}\left[\mathbf{x}_{n}\right]$.

Proof. The proof of Lemma 3.7 shows that the polynomials in question lie in $J_{\alpha, k}$ and Theorem 3.6 guarantees that they can be expressed as $\mathbb{Z}\left[\mathbf{x}_{n}\right]$-linear combinations of the generators of $J_{\alpha, k}$. The result follows from the identification of $\mathcal{M}_{\alpha, k}$ as the standard monomial basis of $S_{\alpha, k}$ in Theorem 3.10 .

\section{MiXed Reduction}

In this section we describe a linear algebra algorithm which will give an affine paving of the Grassmann product $\operatorname{Gr}(\alpha, k)$ which respects the inclusion $X_{\alpha, k} \subseteq G r(\alpha, k)$. Since our algorithm will be a mixture of row and column reduction, we call it mixed reduction. The results in this section are valid over any field $\mathbb{F}$.

We model our moduli spaces using matrices. For any $\alpha \in[k]^{r}$ with $|\alpha|=n$, let $\mathcal{V}_{\alpha}$ be the family of $k \times n$ matrices given in block form by

$$
\mathcal{V}_{\alpha}:=\left\{A=\left(A_{1}|\cdots| A_{r}\right): A_{i} \text { is a } k \times \alpha_{i} \text { matrix and has full rank for } i=1,2, \ldots, r\right\} .
$$

Let $\mathcal{U}_{\alpha} \subseteq \mathcal{V}_{\alpha}$ be the subfamily

$$
\mathcal{U}_{\alpha}:=\left\{A \in \mathcal{V}_{\alpha}: A \text { has full rank }\right\}
$$

of rank $k$ matrices in $\mathcal{V}_{\alpha}$. Both of the sets $\mathcal{V}_{\alpha}$ and $\mathcal{U}_{\alpha}$ carry an action of the Levi subgroup $L_{\alpha} \subseteq G L_{n}$ on their columns. We have the identifications

$$
\mathcal{V}_{\alpha} / L_{\alpha}=\operatorname{Gr}(\alpha, k), \quad \mathcal{U}_{\alpha} / L_{\alpha}=X_{\alpha, k} .
$$


4.1. Permuted column reduced echelon form. We review the classical decomposition of the Grassmannian $\operatorname{Gr}(d, k)$ into Schubert cells. Any point $V \in G r(d, k)$ can be represented as the column space of a full rank $k \times d$ matrix $A$. Applying the action of $G L_{d}$ on columns, we may obtain a unique such representation by putting $A$ into column reduced echelon form (CREF). Given a $d$-element subset $I \subseteq[k]$, the (open) Schubert cell of $G r(d, k)$ consists of all points $V \in G r(d, k)$ represented by matrices whose CREF has pivot row set equal to $I$. For $k=4$ and $d=2$, the Schubert cells are as follows, where the $\star$ 's are arbitrary field elements.

$$
\begin{aligned}
& \left(\begin{array}{ll}
1 & 0 \\
0 & 1 \\
\star & \star \\
\star & \star
\end{array}\right) \quad\left(\begin{array}{ll}
1 & 0 \\
\star & 0 \\
0 & 1 \\
\star & \star
\end{array}\right) \quad\left(\begin{array}{ll}
1 & 0 \\
\star & 0 \\
\star & 0 \\
0 & 1
\end{array}\right) \quad\left(\begin{array}{ll}
0 & 0 \\
1 & 0 \\
0 & 1 \\
\star & \star
\end{array}\right) \quad\left(\begin{array}{ll}
0 & 0 \\
1 & 0 \\
\star & 0 \\
0 & 1
\end{array}\right)\left(\begin{array}{ll}
0 & 0 \\
0 & 0 \\
1 & 0 \\
0 & 1
\end{array}\right) \\
& I=12 \quad I=13 \quad I=14 \quad I=23 \quad I=24 \quad I=34
\end{aligned}
$$

As $I$ varies over all $k$-element subsets of $[n]$, the Schubert cells attached to $I$ induce an affine paving of the Grassmannian $G r(d, k)$. Taking a product of such pavings gives a paving of $G r(\alpha, k)$. Unfortunately, the product paving interacts poorly with the inclusion $X_{\alpha, k} \subseteq G r(\alpha, k)$ : cells $C$ in the product paving can satisfy $C \nsubseteq X_{\alpha, k}$ and $C \cap X_{\alpha, k} \neq \varnothing$ simultaneously. For this reason, we will need a different paving of $\operatorname{Gr}(\alpha, k)$. The first step in describing this new paving is a modification of the column reduction algorithm.

Let $A$ be a matrix with $k$ rows. The usual column reduction algorithm prioritizes the rows of $A$ in the order $1,2, \ldots, k$. If $\pi=\pi(1) \pi(2) \cdots \pi(k) \in \mathfrak{S}_{k}$ is any permutation, we could also prioritize the rows in the order $\pi(1), \pi(2), \ldots, \pi(k)$. Let the output of this algorithm be the $\pi$-column reduced echelon form $(\pi-C R E F)$ of $A$. When $\pi=12 \cdots k$ is the identity permutation, the $\pi$-CREF is the usual CREF. We will only be interested in the case where the permutation $\pi$ has at most one descent.

To see an example of $\pi$-CREF, suppose $k=4, d=2$, and $\pi=2413 \in \mathfrak{S}_{4}$. The possible forms of the $\pi$-CREF of a full rank matrix, together with their pivot sets $I$, are

$$
\begin{aligned}
& \left(\begin{array}{ll}
\star & \star \\
1 & 0 \\
\star & \star \\
0 & 1
\end{array}\right) \quad\left(\begin{array}{ll}
0 & 1 \\
1 & 0 \\
\star & \star \\
\star & 0
\end{array}\right) \quad\left(\begin{array}{ll}
\star & 0 \\
1 & 0 \\
0 & 1 \\
\star & 0
\end{array}\right) \quad\left(\begin{array}{ll}
0 & 1 \\
0 & 0 \\
\star & \star \\
1 & 0
\end{array}\right) \quad\left(\begin{array}{ll}
\star & 0 \\
0 & 0 \\
0 & 1 \\
1 & 0
\end{array}\right)\left(\begin{array}{ll}
1 & 0 \\
0 & 0 \\
0 & 1 \\
0 & 0
\end{array}\right) \\
& I=24 \quad I=12 \quad I=23 \quad I=14 \quad I=34 \quad I=13
\end{aligned}
$$

If $A$ is a $k \times d$ matrix for $d \leq k$ and $I \subseteq[k]$ is a subset of the rows of $A$, let $\Delta_{I}(A)$ be the maximal minor of $A$ with row set $I$. We record an important fact about the entries of a matrix in $\pi$-CREF.

Lemma 4.1. Let $d \leq k$ and let $A$ be a full rank $k \times d$ matrix. Let $\pi \in \mathfrak{S}_{k}$ and let $B$ be the $\pi-C R E F$ of $A$. Suppose that $B$ has pivots in row set $I \subseteq[k]$ (so that $|I|=d$ ). For any position of $(r, s)$ of $B$ such that $r \notin I$, there exists a subset $J=J(r, s) \subseteq[k]$ such that $J$ differs from $I$ in exactly one element and

$$
\text { the }(r, s) \text {-entry of } B \text { is the quotient of maximal minors } \pm \Delta_{J}(A) / \Delta_{I}(A) \text { of } A \text {, }
$$

where the sign depends on $I, r, s$, and $\pi$.

Proof. When $\pi$ is the identity permutation, this is the classical fact that the Plücker coordinates of any point in $\operatorname{Gr}(d, k)$ contain (up to predictable signs and global scaling) the entries in the CREF of a $k \times d$ matrix representing that point. This remains true if we order the columns of our matrices according to an arbitrary permutation $\pi$.

4.2. The mixed reduction algorithm. Let $U \subseteq G L_{k}$ be the subgroup of lower triangular matrices with 1 's on the diagonal. Given a set sequence $\mathcal{I}=\left(I_{1}, \ldots, I_{r}\right)$ in $[k]$, we define a subset $U(\mathcal{I}) \subseteq U$ according to the following sparsity pattern.

Definition 4.2. Let $\mathcal{I}=\left(I_{1}, \ldots, I_{r}\right)$ be a set sequence in $[k]$. Let $U(\mathcal{I})$ consist of those matrices $\left(u_{i, j}\right)_{1 \leq i, j \leq k} \in U$ such that for $1 \leq j<i \leq k$ we have 
$u_{i, j}=0$ unless there exists $1 \leq t \leq n$ such that $i \in I_{t}-\left(I_{1} \cup \cdots \cup I_{t-1}\right)$ and $j \notin\left(I_{1} \cup \cdots \cup I_{t-1} \cup I_{t}\right)$.

When $k=4$, two examples of $U(\mathcal{I})$ - one for a covering set sequence and one for a non-covering set sequence - are as follows:

$$
U(24,34,3,14)=\left(\begin{array}{cccc}
1 & 0 & 0 & 0 \\
0 & 1 & 0 & 0 \\
0 & \star & 1 & 0 \\
0 & 0 & 0 & 1
\end{array}\right) \quad U(14,12,1,12)=\left(\begin{array}{cccc}
1 & 0 & 0 & 0 \\
\star & 1 & 0 & 0 \\
\star & \star & 1 & 1 \\
0 & 0 & 0 & 1
\end{array}\right)
$$

The set $U(\mathcal{I})$ is a copy of affine space of dimension equal to the number of $\star$ 's.

Given a set sequence $\mathcal{I}=\left(I_{1}, \ldots, I_{r}\right)$ in $[k]$, we also define a sequence of $r$ permutations in $\mathfrak{S}_{k}$.

Definition 4.3. Let $\mathcal{I}=\left(I_{1}, \ldots, I_{r}\right)$ be set sequence in $[k]$. Define $r$ permutations $\pi_{\mathcal{I}}^{(1)}, \ldots, \pi_{\mathcal{I}}^{(r)} \in$ $\mathfrak{S}_{k}$ as follows. The one-line notation $\pi_{\mathcal{I}}^{(t)}$ is obtained by listing the elements of $[k]-\left(I_{1} \cup \cdots \cup I_{t-1}\right)$ in increasing order, then writing the elements of $I_{1} \cup \cdots \cup I_{t-1}$ in increasing order. We write $\pi^{(t)}$ instead of $\pi_{\mathcal{I}}^{(t)}$ when the set sequence is understood.

If $\mathcal{I}=(24,34,3,14)$ we get the permutation sequence $\pi^{(1)}=1234, \pi^{(2)}=1324, \pi^{(3)}=1234$, and $\pi^{(4)}=1234$. For our other example $\mathcal{I}=(14,12,1,12)$ we get the permutation sequence $\pi^{(1)}=1234, \pi^{(2)}=2314, \pi^{(3)}=3124$, and $\pi^{(4)}=3124$.

Both the affine space $U(\mathcal{I})$ and the permutation sequence $\pi_{\mathcal{I}}^{(1)}, \ldots, \pi_{\mathcal{I}}^{(r)}$ only depend on the initial unions of the sets in $\mathcal{I}$.

Observation 4.4. Let $\mathcal{I}=\left(I_{1}, \ldots I_{r}\right)$ and $\mathcal{I}^{\prime}=\left(I_{1}^{\prime}, \ldots, I_{r}^{\prime}\right)$ be two set sequences in $[k]$ of the same type. Suppose that $I_{1} \cup \cdots \cup I_{t}=I_{t}^{\prime} \cup \cdots \cup I_{t}^{\prime}$ for each $1 \leq t \leq r$. Then $U(\mathcal{I})=U\left(\mathcal{I}^{\prime}\right)$ and $\pi_{\mathcal{I}}^{(t)}=\pi_{\mathcal{I}^{\prime}}^{(t)}$ for each $1 \leq t \leq r$.

The unipotent group $U \subseteq G L_{k}$ and its subsets $U(\mathcal{I})$ act on the rows of matrices in $\mathcal{V}_{\alpha}$ and $\mathcal{U}_{\alpha}$. Mixed reduction starts with a matrix $A \in \mathcal{V}_{\alpha}$ and outputs a set sequence $\mathcal{I}$ of type $\alpha$, a matrix $u \in U(\mathcal{I})$, and a matrix $B \in \mathcal{V}_{\alpha, k}$ fitting a certain sparsity pattern determined by $\mathcal{I}$ such that $A=u B g$ for some element $g \in L_{\alpha}$. We describe the pattern satisfied by the output matrix $B$.

Definition 4.5. Let $\mathcal{I}=\left(I_{1}, \ldots, I_{r}\right)$ be a set sequence of type $\alpha \in[k]^{r}$ where $|\alpha|=n$. Let $\pi^{(1)}, \ldots, \pi^{(r)} \in \mathfrak{S}_{k}$ be the permutation sequence associated to $\mathcal{I}$. The pattern matrix $\mathrm{PM}(\mathcal{I})$ is a $k \times n$ matrix over the set $\{0,1, \star\}$ with block form $\operatorname{PM}(\mathcal{I})=\left(A_{1}|\cdots| A_{r}\right)$ where $A_{t}$ is $k \times \alpha_{t}$. For $1 \leq t \leq r$, the block $A_{t}$ is obtained as follows.

Let $C_{t}$ be the $\{0,1, \star\}$-matrix describing $\pi^{(t)}$-CREF with pivot set $I_{t}$. For any $j>i$ such that $i \in I_{t}-\left(I_{1} \cup \cdots \cup I_{t-1}\right)$ and $j \in[k]-\left(I_{1} \cup \cdots \cup I_{t-1} \cup I_{t}\right)$, there will be a $\star$ in $C_{t}$ in row $j$ below the pivot in row $i$. The block $A_{t}$ of $\mathrm{PM}(\mathcal{I})$ is obtained from $C_{t}$ by replacing all such 's with 0's.

We give the pattern matrices corresponding to our two example set sequences. If $\mathcal{I}=(24,34,3,14)$ the associated pattern matrix is

$$
\operatorname{PM}(24,34,3,13)=\left(\begin{array}{cc|cc|c|cc}
0 & 0 & 0 & 0 & 0 & 1 & 0 \\
1 & 0 & \star & 0 & 0 & \star & 0 \\
0 & 0 & 1 & 0 & 1 & 0 & 1 \\
0 & 1 & 0 & 1 & \star & \star & \star
\end{array}\right)
$$

The $(3,1)$-entry of the first block is 0 instead of $\star$ because of the second paragraph of Definition 4.5 . If $\mathcal{I}=(14,12,1,12)$ the associated pattern matrix is

$$
\operatorname{PM}(14,12,1,12)=\left(\begin{array}{cc|cc|c|cc}
1 & 0 & 0 & 1 & 1 & 1 & 0 \\
0 & 0 & 1 & 0 & \star & 0 & 1 \\
0 & 0 & 0 & 0 & 0 & 0 & 0 \\
0 & 1 & \star & \star & \star & \star & \star
\end{array}\right)
$$

The $(2,1)$ - and $(3,1)$-entries of the first block and the $(3,1)$-entry of the second block are 0 instead of $\star$ because of the second paragraph of Definition 4.5, 
A pattern matrix $\operatorname{PM}(\mathcal{I})$ has a zero row if and only if the set sequence $\mathcal{I}$ fails to cover $[k]$. For any set sequence $\mathcal{I}$, a matrix $B \in \mathcal{V}_{\alpha}$ is said to fit the pattern of $\mathcal{I}$ if $B$ can be obtained by replacing the $\star$ 's in $\operatorname{PM}(\mathcal{I})$ with field elements.

\section{MIXED REDUCTION ALGORITHM}

INPUT: Integers $k, r>0$, a length $r$ sequence $\alpha=\left(\alpha_{1}, \ldots, \alpha_{r}\right) \in[k]^{r}$, and a matrix $A \in \mathcal{V}_{\alpha}$

OUTPUT: A set sequence $\mathcal{I}=\left(I_{1}, \ldots, I_{r}\right)$ in $[k]$ of type $\alpha$, an element $u \in U(\mathcal{I})$, and a matrix $B \in \mathcal{V}_{\alpha}$ which fits the pattern of $\mathcal{I}$ such that $A=u B g$ for some element $g \in L_{\alpha}$

(1) Initialize a block matrix $B:=\left(B_{1}|\cdots| B_{r}\right)$ by $B_{t}:=A_{t}$. Initialize $\mathcal{I}$ to be the empty set sequence. Initialize $u$ to be the $k \times k$ identity matrix.

(2) For $t=1,2, \ldots, r$ do the following,

(a) Let $\pi^{(t)}=\pi^{(t)}(1) \ldots \pi^{(t)}(k)$ be the permutation in $\mathfrak{S}_{k}$ obtained by writing the elements of $[k]-\left(I_{1} \cup \cdots \cup I_{t-1}\right)$ in increasing order, and then writing the elements of $I_{1} \cup \cdots \cup I_{t-1}$ in increasing order.

(b) Use column operations on $B_{t}$ to put $B_{t}$ into $\pi^{(t)}$-CREF. Let $I_{t} \subseteq[k]$ be the set of pivot rows obtained thereby. Append $I_{t}$ to the set sequence $\mathcal{I}$.

(c) For any $j>i$ such that $i \in I_{t}-\left(I_{1} \cup \cdots \cup I_{t-1}\right)$ and $j \in[k]-\left(I_{1} \cup \cdots \cup I_{t}\right)$, modify the $(j, i)$-entry of $u$ to clear the element of $B_{t}$ in row $j$ below the pivot in row $i$. This performs a row operation on the overall matrix $B$.

We consider two examples of the mixed reduction algorithm.

Example 4.6. Take $k=4, \alpha=(2,2,1,2)$, and start with the matrix

$$
A=\left(\begin{array}{cc|cc|c|cc}
0 & 0 & 1 & 0 & 4 & 1 & 1 \\
1 & 0 & -1 & 0 & -2 & 2 & 2 \\
-1 & 0 & 2 & 0 & 8 & -1 & 0 \\
0 & 1 & 0 & 1 & 4 & -1 & -1
\end{array}\right)
$$

We process the blocks of $A$ from left to right:

$$
\begin{aligned}
&\left(\begin{array}{cc|cc|c|cc}
0 & 0 & 1 & 0 & 4 & 1 & 1 \\
1 & 0 & -1 & 0 & -2 & 2 & 2 \\
-1 & 0 & 2 & 0 & 8 & -1 & 0 \\
0 & 1 & 0 & 1 & 4 & -1 & -1
\end{array}\right) \rightarrow\left(\begin{array}{cc|cc|c|ccc}
0 & 0 & 1 & 0 & 4 & 1 & 1 \\
1 & 0 & -1 & 0 & -2 & 2 & 2 \\
0 & 0 & 1 & 0 & 6 & 1 & 2 \\
0 & 1 & 0 & 1 & 4 & -1 & -1
\end{array}\right) \rightarrow\left(\begin{array}{cc|cc|c|cc}
0 & 0 & 1 & 0 & 4 & 1 & 1 \\
1 & 0 & -1 & 0 & -2 & 2 & 2 \\
0 & 0 & 0 & 0 & 2 & 0 & 1 \\
0 & 1 & 0 & 1 & 4 & -1 & -1
\end{array}\right) \rightarrow \\
&\left(\begin{array}{cc|cc|c|ccc}
0 & 0 & 1 & 0 & 2 & 1 & 1 \\
1 & 0 & -1 & 0 & -1 & 2 & 2 \\
0 & 0 & 0 & 0 & 1 & 0 & 1 \\
0 & 1 & 0 & 1 & 2 & -1 & -1
\end{array}\right) \rightarrow\left(\begin{array}{cc|cc|c|cc}
0 & 0 & 1 & 0 & 2 & 1 & 0 \\
1 & 0 & -1 & 0 & -1 & 2 & 0 \\
0 & 0 & 0 & 0 & 1 & 0 & 1 \\
0 & 1 & 0 & 1 & 2 & -1 & 2
\end{array}\right)
\end{aligned}
$$

More explicitly, the steps are

(1) The first block of $A$ is already in $\pi^{(1)}-C R E F$ where $\pi^{(1)}=1234$. We see that $I_{1}=24$.

(2) We use the pivot in row 2 to cancel row 3.

(3) The second block of $A$ is already in $\pi^{(2)}$ - CREF where $\pi^{(2)}=1324$. We see that $I_{2}=14$.

(4) We use the pivot in row 1 to cancel row 3.

(5) We find the $\pi^{(3)}$-CREF of the third block of $A$ where $\pi^{(3)}=3124$. We see that $I_{3}=3$.

(6) We find the $\pi^{(4)}$-CREF of the fourth block of $A$ where $\pi^{(4)}=1234$. We see that $I_{4}=13$.

(7) We use the pivot in row 1 to cancel rows 2 and 4.

The output set sequence $\mathcal{I}=\left(I_{1}, I_{2}, I_{3}, I_{4}\right)$ is $(24,14,3,13)$. The unipotent subset $U(\mathcal{I})$ and the matrix $u \in U(\mathcal{I})$ are

$$
U(\mathcal{I})=\left(\begin{array}{llll}
1 & 0 & 0 & 0 \\
0 & 1 & 0 & 0 \\
\star & \star & 1 & 0 \\
0 & 0 & 0 & 1
\end{array}\right) \quad u=\left(\begin{array}{cccc}
1 & 0 & 0 & 0 \\
0 & 1 & 0 & 0 \\
1 & -1 & 1 & 0 \\
0 & 0 & 0 & 1
\end{array}\right)
$$


Example 4.7. Our second example applies mixed reduction to a matrix whose rank is not full. Let

$$
A=\left(\begin{array}{cc|cc|c|cc}
2 & 0 & 0 & 1 & -1 & 1 & 1 \\
2 & 0 & 1 & 1 & 0 & 3 & 3 \\
-2 & 0 & 1 & -1 & 2 & 1 & 1 \\
1 & 1 & 2 & 2 & -1 & -1 & 0
\end{array}\right)
$$

Applying mixed reduction to A yields the following.

$$
\begin{aligned}
& \left(\begin{array}{cc|cc|c|cc}
2 & 0 & 0 & 1 & -1 & 1 & 1 \\
2 & 0 & 1 & 1 & 0 & 3 & 3 \\
-2 & 0 & 1 & -1 & 2 & 1 & 1 \\
1 & 1 & 2 & 2 & -1 & -1 & 0
\end{array}\right) \rightarrow\left(\begin{array}{cc|cc|c|cc}
1 & 0 & 0 & 1 & -1 & 1 & 1 \\
1 & 0 & 1 & 1 & 0 & 3 & 3 \\
-1 & 0 & 1 & -1 & 2 & 1 & 1 \\
0 & 1 & 2 & 2 & -1 & -1 & 0
\end{array}\right) \rightarrow\left(\begin{array}{cc|cc|c|cc}
1 & 0 & 0 & 1 & -1 & 1 & 1 \\
0 & 0 & 1 & 0 & 1 & 2 & 2 \\
0 & 0 & 1 & 0 & 1 & 2 & 2 \\
0 & 1 & 2 & 2 & -1 & -1 & 0
\end{array}\right) \rightarrow \\
& \left(\begin{array}{cc|cc|c|cc}
1 & 0 & 0 & 1 & -1 & 1 & 1 \\
0 & 0 & 1 & 0 & 1 & 2 & 2 \\
0 & 0 & 0 & 0 & 0 & 0 & 0 \\
0 & 1 & 2 & 2 & -1 & -1 & 0
\end{array}\right) \rightarrow\left(\begin{array}{cc|cc|c|cc}
1 & 0 & 0 & 1 & 1 & 1 & 1 \\
0 & 0 & 1 & 0 & -1 & 2 & 2 \\
0 & 0 & 0 & 0 & 0 & 0 & 0 \\
0 & 1 & 2 & 2 & 1 & -1 & 0
\end{array}\right) \rightarrow\left(\begin{array}{cc|cc|c|cc}
1 & 0 & 0 & 1 & 1 & 1 & 0 \\
0 & 0 & 1 & 0 & -1 & 2 & 0 \\
0 & 0 & 0 & 0 & 0 & 0 & 0 \\
0 & 1 & 2 & 2 & 1 & 0 & 1
\end{array}\right)
\end{aligned}
$$

More explicitly, the steps are

(1) We find the $\pi^{(1)}-C R E F$ of the first block of $A$ where $\pi^{(1)}=1234$. We see that $I_{1}=14$.

(2) We use the pivot in row 1 to cancel rows 2 and 3.

(3) The second block of $A$ is already in $\pi^{(2)}-C R E F$ where $\pi^{(2)}=2314$. We see that $I_{2}=12$.

(4) We use the pivot in row 2 to cancel row 3.

(5) We find the $\pi^{(3)}$-CREF of the third block of $A$ where $\pi^{(3)}=3124$. We see that $I_{3}=1$.

(6) We find the $\pi^{(4)}$-CREF of the fourth block of $A$ where $\pi^{(4)}=3124$. We see that $I_{4}=14$.

The output set sequence $\mathcal{I}=\left(I_{1}, I_{2}, I_{3}, I_{4}\right)$ is $(14,12,1,14)$. The unipotent subeset $U(\mathcal{I})$ and the matrix $u \in U(\mathcal{I})$ are

$$
U(\mathcal{I})=\left(\begin{array}{llll}
1 & 0 & 0 & 0 \\
\star & 1 & 0 & 0 \\
\star & \star & 1 & 0 \\
0 & 0 & 0 & 1
\end{array}\right) \quad u=\left(\begin{array}{cccc}
1 & 0 & 0 & 0 \\
-1 & 1 & 0 & 0 \\
1 & 1 & 1 & 0 \\
0 & 0 & 0 & 1
\end{array}\right) .
$$

Observe that once the mixed reduction algorithm processes the $i^{\text {th }}$ block of a matrix, that block remains unchanged for the remainder of the algorithm: any row operations used for later blocks will involve two rows of block $i$, the higher of which is a row of zeroes. The following linear algebra result is the foundation of our nonstandard affine paving of $\operatorname{Gr}(\alpha, k)$.

Proposition 4.8. Let $A \in \mathcal{V}_{\alpha}$. There exists a unique set sequence $\mathcal{I}$ in $[k]$ of type $\alpha$, a unique matrix $u \in U(\mathcal{I})$, a unique matrix $B$ fitting the pattern of $\mathcal{I}$, and a unique Levi element $g \in L_{\alpha}$ such that $A=u B g$. We have $A \in \mathcal{U}_{\alpha}$ if and only if the set sequence $\mathcal{I}$ covers $[k]$.

Proof. Existence comes from applying the mixed reduction algorithm to the matrix $A$. Uniqueness of $g$ comes from the fact that the blocks $A=\left(A_{1}|\cdots| A_{r}\right)$ of $A$ have full rank. Uniqueness of $u, B$, and $\mathcal{I}$ follow from uniqueness of the $\pi$-CREF for any permutation $\pi \in \mathfrak{S}_{k}$. The last sentence is true because $B$ is nonsingular if and only if $\mathcal{I}$ covers $[k]$.

4.3. Geometric consequences of mixed reduction. Given a set sequence $\mathcal{I}$, we define two subsets $C_{\mathcal{I}}$ and $D_{\mathcal{I}}$ of the Grassmann product as follows.

Definition 4.9. Let $\alpha \in[k]^{r}$ and let $\mathcal{I}$ be a set sequence in $[k]$ of type $\alpha$. Define two subsets $\widehat{D_{\mathcal{I}}} \subseteq \widehat{C_{\mathcal{I}}} \subseteq \mathcal{V}_{\alpha}$ by

$$
\begin{aligned}
& \widehat{C_{\mathcal{I}}}:=\{u B: u \in U(\mathcal{I}) \text { and } B \text { fits the pattern of } \mathcal{I}\} . \\
& \widehat{D_{\mathcal{I}}}:=\{B: B \text { fits the pattern of } \mathcal{I}\} .
\end{aligned}
$$

Define $D_{\mathcal{I}} \subseteq C_{\mathcal{I}} \subseteq G r(\alpha, k)$ by

$$
\begin{aligned}
& C_{\mathcal{I}}:=\text { image of } \widehat{C_{\mathcal{I}}} \text { in } \operatorname{Gr}(\alpha, k) . \\
& D_{\mathcal{I}}:=\text { image of } \widehat{D_{\mathcal{I}}} \text { in } \operatorname{Gr}(\alpha, k) .
\end{aligned}
$$


The sets $C_{\mathcal{I}}$ and $D_{\mathcal{I}}$ are related by $C_{\mathcal{I}}=U(\mathcal{I}) D_{\mathcal{I}}$. A more precise relationship is as follows.

Lemma 4.10. Let $\alpha \in[k]^{r}$ and let $\mathcal{I}=\left(I_{1}, \ldots, I_{r}\right)$ be a set sequence in $[k]$ of type $\alpha$.

(1) The subset $D_{\mathcal{I}} \subseteq G r(\alpha, k)$ is isomorphic to affine space of dimension equal to the number of $\star$ 's in the pattern matrix $\operatorname{PM}(\mathcal{I})$.

(2) Matrix multiplication gives an isomorphism of varieties $\widehat{C_{\mathcal{I}}} L_{\alpha} \cong U(\mathcal{I}) \times \widehat{D_{\mathcal{I}}} L_{\alpha}$.

(3) Matrix multiplication induces an isomorphism of varieties $C_{\mathcal{I}} \cong U(\mathcal{I}) \times D_{\mathcal{I}}$.

(4) The subset $C_{\mathcal{I}} \subseteq G r(\alpha, k)$ is isomorphic to affine space of dimension equal to the number of $\star$ 's in the pattern matrix $\mathrm{PM}(\mathcal{I})$ plus the dimension of the affine space $U(\mathcal{I})$.

Proof. (1) It is clear that $\widehat{D_{\mathcal{I}}}$ is a copy of affine space of the desired dimension. By Proposition 4.8 , the canonical projection $\widehat{D_{\mathcal{I}}} \rightarrow D_{\mathcal{I}}$ is a regular bijection. We need only check that the inverse function $D_{\mathcal{I}} \rightarrow \widehat{D_{\mathcal{I}}}$ is regular.

The regularity of $D_{\mathcal{I}} \rightarrow \widehat{D_{\mathcal{I}}}$ is a consequence of Lemma 4.1, Let $\pi^{(1)}, \ldots, \pi^{(r)} \in \mathfrak{S}_{k}$ be the permutation sequence corresponding to $\mathcal{I}$ and let $A=\left(A_{1}|\cdots| A_{r}\right) \in \widehat{D_{\mathcal{I}}}$. For each $1 \leq t \leq r$, the matrix $A_{t}$ is in $\pi^{(t)}$ CREF with pivot row set $I_{t}$. The rows of $A$ in $I_{t}$ are determined by $\pi^{(t)}$. Lemma 4.1 says that the other entries of $A$ are images under regular functions on $D_{\mathcal{I}}$ of the form $\pm \Delta_{J}^{(t)} / \Delta_{I_{t}}^{(t)}$, where $|J|=\left|I_{t}\right|$, the sets $J$ and $I_{t}$ differ in a single element, the sign is deterministic, and the superscript indicates that we are taking Plücker coordinates in the $t^{\text {th }}$ factor of $D_{\mathcal{I}}$.

(2) Matrix multiplication gives a regular map $\mu: U(\mathcal{I}) \times \widehat{D_{\mathcal{I}}} L_{\alpha} \rightarrow \widehat{C_{\mathcal{I}}} L_{\alpha}$. Proposition 4.8 tells us that $\mu$ is a bijection. We need only show that the inverse map $\mu^{-1}: \widehat{C_{\mathcal{I}}} L_{\alpha} \rightarrow U(\mathcal{I}) \times \widehat{D_{\mathcal{I}}} L_{\alpha}$ is regular.

Let $u \in U(\mathcal{I})$ and let $A=\left(A_{1}|\cdots| A_{r}\right) \in \widehat{D_{\mathcal{I}}} L_{\alpha}$ so that $u A=B=\left(B_{1}|\cdots| B_{r}\right) \in \widehat{C_{\mathcal{I}}} L_{\alpha}$. We need to show that the entries of $u$ and $A$ are regular functions of the entries of $B$.

Consider applying mixed reduction to the matrix $B$ to get a matrix pair $u^{\prime} \in U(\mathcal{I})$ and $A^{\prime} \in \widehat{D_{\mathcal{I}}}$. To process the first block, we find the unique $g_{1} \in G L_{\alpha_{1}}$ such that $B_{1} g_{1}$ is in $\pi^{(1)}$-CREF. The matrix $g_{1}$ is a regular function of the entries of $B_{1}$ (the rows of $g_{1}^{-1}$ are the maximal submatrix of $B_{1}$ with row set $I_{1}$ ). Next, we fill in the nonzero entries in columns $I_{1}$ of $u^{\prime}$ with entries of $B_{1} g_{1}$ (which are regular functions of $B$ ) and apply the corresponding row operations to $B$ to get a matrix $B^{\prime}=\left(A_{1}^{\prime}\left|B_{2}^{\prime}\right| \cdots \mid B_{r}^{\prime}\right)$ (whose entries are regular functions of $B$ ) and whose first block coincides with the first block of $A^{\prime}$. Iterating, we get a matrix $g=g_{1} \oplus \cdots \oplus g_{t}$ in $L_{\alpha}$ such that $B=u^{\prime} A^{\prime} g$, where each of $u^{\prime}, A$, and $g$ are regular functions of $B$.

Since $B=u^{\prime} A^{\prime} g=u A$ and $A \in \widehat{D_{\mathcal{I}}} L_{\alpha}$, Proposition 4.8 tells us that $u=u^{\prime}$ and $A^{\prime} g=A$. It follows that the entries of $u$ are regular functions of $B$. Since the entries of both $A^{\prime}$ and $g$ are regular functions of $B$, so are the entries of $A=A^{\prime} g$.

(3) and (4) both follow immediately from (2).

The cells $C_{\mathcal{I}}$ of Lemma 4.10 will induce our affine paving of $\operatorname{Gr}(\alpha, k)$ which interacts nicely with the inclusion $X_{\alpha, k} \subseteq \operatorname{Gr}(\alpha, k)$. The set-theoretic result in this direction is as follows.

Lemma 4.11. Let $\alpha \in[k]^{r}$. We have a disjoint union decomposition

$$
G r(\alpha, k)=\bigsqcup_{\mathcal{I}} C_{\mathcal{I}}
$$

where $\mathcal{I}$ ranges over the collection of all set sequences $\left(I_{1}, \ldots, I_{r}\right)$ of type $\alpha$ in $[k]$. For a given set sequence $\mathcal{I}$, we have $C_{\mathcal{I}} \subseteq X_{\alpha, k}$ if $\mathcal{I}$ covers $[k]$ and $C_{\mathcal{I}} \cap X_{\alpha, k}=\varnothing$ otherwise.

Proof. The disjoint union decomposition follows from Proposition 4.8, Let $\mathcal{I}$ be a set sequence and suppose that $B$ fits the pattern of $\mathcal{I}$. From the definition of pattern matrices we see that $B \in \mathcal{U}_{\alpha}$ if and only if the set sequence $\mathcal{I}$ covers $[k]$, which verifies the second sentence of the lemma.

The final goal of this section is to show that the cells $C_{\mathcal{I}}$ stratifying $\operatorname{Gr}(\alpha, k)$ fit together nicely. 
Theorem 4.12. Let $\alpha \in[k]^{r}$ with $|\alpha|=n$. The cells

$$
\left\{C_{\mathcal{I}}: \mathcal{I}=\left(I_{1}, \ldots, I_{r}\right) \text { is a set sequence in }[k] \text { of type } \alpha\right\}
$$

induce an affine paving of the Grassmann product $G r(\alpha, k)$. Furthermore, this affine paving

$$
\varnothing=X_{0} \subset X_{1} \subset \cdots \subset X_{m}=G r(\alpha, k)
$$

may be chosen in such a way that $X_{\alpha, k}=G r(\alpha, k)-X_{i}$ for some $i$.

Proof. By Lemma 4.11 we know that the $C_{\mathcal{I}}$ are cells whose disjoint union is $\operatorname{Gr}(\alpha, k)$. In order to show that they induce an affine paving of $G r(\alpha, k)$, it remains to demonstrate that they may be totally ordered in such a way that all initial cell unions are Zariski closed in $\operatorname{Gr}(\alpha, k)$.

Given any $k \times n$ matrix $A$ and any position $(i, j) \in[k] \times[n]$, we define a number

$$
\operatorname{rk}(i, j, A):=\operatorname{rank} \text { of the northwest } i \times j \text { submatrix of } A \text {. }
$$

If $u \in U \subseteq G L_{k}$ is any lower triangular $k \times k$ unipotent matrix, we have

$$
\operatorname{rk}(i, j, u A)=\operatorname{rk}(i, j, A)
$$

since we are taking northwest submatrices. Furthermore, for any $1 \leq j \leq n$ we have the growth conditions

$$
\operatorname{rk}(i-1, j, A) \leq \operatorname{rk}(i, j, A) \leq \operatorname{rk}(i-1, j, A)+1, \quad i=1,2, \ldots, k
$$

where we adopt the convention $\operatorname{rk}(0, j, A)=0$.

The function $\operatorname{rk}(i, j,-)$ is well-defined on $\operatorname{Gr}(\alpha, k)$ whenever $j \in\left\{\alpha_{1}, \alpha_{1}+\alpha_{2}, \ldots\right\}$. We have the disjoint union decomposition

$$
\operatorname{Gr}(\alpha, k)=\bigsqcup_{\rho} \Omega_{\rho}
$$

where $\rho$ varies over all functions $\rho:[k] \times\left\{\alpha_{1}, \alpha_{1}+\alpha_{2}, \ldots\right\} \rightarrow \mathbb{Z}_{\geq 0}$ and $\Omega_{\rho}$ is given by

$$
\Omega_{\rho}:=\left\{[A] \in G r(\alpha, k): \operatorname{rk}(i, j, A)=\rho(i, j) \text { for all } 1 \leq i \leq k \text { and } j \in\left\{\alpha_{1}, \alpha_{1}+\alpha_{2}, \ldots\right\}\right\},
$$

where $A \in \mathcal{V}_{\alpha}$ represents the point $[A] \in G r(\alpha, k)$. Although the indexing set of the decomposition (4.12) is infinite, all but finitely many of the sets $\Omega_{\rho}$ are empty.

If we totally order the sets $\Omega_{\rho}$ in the decomposition (4.12) in an extension of the partial order on functions $\rho$ given by $\rho \leq \rho^{\prime}$ if and only if $\rho(i, j) \leq \rho^{\prime}(i, j)$ for all $i, j$, then any initial union of the $\Omega_{\rho}$ will be Zariski closed in $\operatorname{Gr}(\alpha, k)$. The idea is to show that, for fixed $\rho$, the set $\Omega_{\rho}$ is isomorphic to a product of affine spaces and Grassmannians, and that appropriate cells $C_{\mathcal{I}}$ cut out a Schubert-type decomposition of this product. To see what the $\Omega_{\rho}$ look like, we give a more combinatorial version of the decomposition (4.12).

Let $\rho:[k] \times\left\{\alpha_{1}, \alpha_{1}+\alpha_{2}, \ldots\right\} \rightarrow \mathbb{Z}_{\geq 0}$ be such that $\Omega_{\rho} \neq \varnothing$. We have the slow growth condition

$$
\rho(i-1, j) \leq \rho(i, j) \leq \rho(i-1, j)+1,
$$

where we set $\rho(0, j):=0$. This motivates the jump sets $J_{t}(\rho) \subseteq[k]$ given by

$$
J_{t}(\rho):=\left\{1 \leq i \leq k: \rho\left(i-1, \alpha_{1}+\cdots+\alpha_{t}\right)<\rho\left(i, \alpha_{1}+\cdots+\alpha_{t}\right)\right\}, \quad 1 \leq t \leq r .
$$

The function $\rho$ can be recovered from the jump sets $\left(J_{1}(\rho), \ldots, J_{r}(\rho)\right)$. Since $\Omega_{\rho} \neq \varnothing$, these jump sets satisfy the containments

$$
J_{1}(\rho) \subseteq J_{2}(\rho) \subseteq \cdots \subseteq J_{r}(\rho)
$$

as well as the size conditions

$$
\max \left(\alpha_{i},\left|J_{i-1}(\rho)\right|\right) \leq\left|J_{i}(\rho)\right| \leq\left|J_{i-1}(\rho)\right|+\alpha_{i},
$$

where we set $J_{0}(\rho):=\varnothing$. We say that a length $r$ sequence $\mathcal{J}=\left(J_{1}, \ldots, J_{r}\right)$ of subsets of $[k]$ is a jump sequence if it satisfies the conditions (4.16) and (4.17). If we let $\Omega_{\mathcal{J}}$ be the family of 
points in $\operatorname{Gr}(\alpha, k)$ whose rank function $\rho$ has jump set sequence $\mathcal{J}$, we have the following finite and irredundant version of the decomposition 4.12 .

$$
\operatorname{Gr}(\alpha, k)=\bigsqcup_{\mathcal{J}} \Omega_{\mathcal{J}}
$$

where the union is over all jump sequences $\mathcal{J}$.

Fix a jump sequence $\mathcal{J}=\left(J_{1}, \ldots, J_{r}\right)$ for the remainder of the proof and consider the piece $\Omega_{\mathcal{J}}$ of the decomposition (4.18). Let $\mathcal{I}=\left(I_{1}, \ldots, I_{r}\right)$ be a set sequence in $[k]$ of type $\alpha$ and consider the associated cell $C_{\mathcal{I}}$. These two subsets of $\operatorname{Gr}(\alpha, k)$ are related by the following dichotomy.

$$
\text { If } I_{1} \cup \cdots \cup I_{i}=J_{i} \text { for all } i=1,2, \ldots, r \text { then } C_{\mathcal{I}} \subseteq \Omega_{\mathcal{J}} \text {. Otherwise } C_{\mathcal{I}} \cap \Omega_{\mathcal{J}}=\varnothing \text {. }
$$

This dichotomy comes from the fact that if $A$ is any matrix which fits the pattern of $\mathcal{I}$, then

$$
\operatorname{rk}\left(i, \alpha_{1}+\cdots+\alpha_{t}, A\right)=\text { number of 1's northwest of }\left(i, \alpha_{1}+\cdots+\alpha_{t}\right) \text { in } \operatorname{PM}(\mathcal{I}) .
$$

We have the disjoint union decomposition

$$
\Omega_{\mathcal{J}}=\bigsqcup_{\mathcal{I}} C_{\mathcal{I}},
$$

where $\mathcal{I}$ ranges over all set sequences $\mathcal{I}=\left(I_{1}, \ldots, I_{r}\right)$ in $[k]$ of type $\alpha$ such that $I_{1} \cup \cdots \cup I_{i}=J_{i}$ for $i=1,2, \ldots, r$; call such set sequences $\mathcal{J}$-compatible.

By Observation 4.4, for any two $\mathcal{J}$-compatible set sequences $\mathcal{I}, \mathcal{I}^{\prime}$ we have $\pi_{\mathcal{I}}^{(t)}=\pi_{\mathcal{I}^{\prime}}^{(t)}$ for all $1 \leq t \leq r$ and $U(\mathcal{I})=U\left(\mathcal{I}^{\prime}\right)$. We therefore let $\pi^{(1)}, \ldots, \pi^{(t)} \in \mathfrak{S}_{k}$ and $U(\mathcal{J})$ be the permutation sequence and unipotent matrix set associated to any $\mathcal{J}$-compatible set sequence $\mathcal{I}$.

We have a bijection induced by matrix multiplication

$$
\Omega_{\mathcal{J}}=\bigsqcup_{\mathcal{I} \text { is }} \bigsqcup_{\mathcal{J} \text {-compatible }} C_{\mathcal{I}} \cong U(\mathcal{J}) \times \bigsqcup_{\mathcal{I} \text { is } \mathcal{J} \text {-compatible }} D_{\mathcal{I}}
$$

where $C_{\mathcal{I}} \cong U(\mathcal{J}) \times D_{\mathcal{I}}$ by Lemma 4.10 (3). An argument similar to that of Lemma 4.10 shows that the bijection (4.21) is an isomorphism of varieties.

The cells $D_{\mathcal{I}}$ for $\mathcal{J}$-compatible set sequences $\mathcal{I}$ induce an affine paving of the disjoint union $\bigsqcup_{\mathcal{I}}$ is $\mathcal{J}$-compatible $D_{\mathcal{I}}$ forming the second factor of Equation (4.21). This affine paving is best understood with an example. Suppose $k=4, \alpha=(2,2,2)$, and $\mathcal{J}=(13,134,134)$. There are six $\mathcal{J}$-compatible set sequences $\mathcal{I}=\left(I_{1}, I_{2}, I_{3}\right)$ of type $\alpha$. The corresponding cells $D_{\mathcal{I}}$ are displayed below.

$$
\begin{gathered}
\left(\begin{array}{ll|ll|ll}
1 & 0 & \star & 0 & 0 & 0 \\
0 & 0 & 0 & 0 & 0 & 0 \\
0 & 1 & 0 & 1 & 1 & 0 \\
0 & 0 & 1 & 0 & 0 & 1
\end{array}\right)\left(\begin{array}{ll|ll|ll}
1 & 0 & 0 & 1 & 0 & 0 \\
0 & 0 & 0 & 0 & 0 & 0 \\
0 & 1 & \star & \star & 1 & 0 \\
0 & 0 & 1 & 0 & 0 & 1
\end{array}\right)\left(\begin{array}{cc|cc|cc}
1 & 0 & \star & 0 & 1 & 0 \\
0 & 0 & 0 & 0 & 0 & 0 \\
0 & 1 & 0 & 1 & \star & 0 \\
0 & 0 & 1 & 0 & 0 & 1
\end{array}\right) \\
\mathcal{I}=(13,34,34) \\
\mathcal{I}=(13,14,34) \\
\left(\begin{array}{ll|ll|ll}
1 & 0 & 0 & 1 & 1 & 0 \\
0 & 0 & 0 & 0 & 0 & 0 \\
0 & 1 & \star & \star & \star & 0 \\
0 & 0 & 1 & 0 & 0 & 1
\end{array}\right) \quad\left(\begin{array}{ll|ll|ll}
1 & 0 & \star & 0 & 1 & 0 \\
0 & 0 & 0 & 0 & 0 & 0 \\
0 & 1 & 0 & 1 & 0 & 1 \\
0 & 0 & 1 & 0 & \star & \star
\end{array}\right) \quad\left(\begin{array}{cc|cc|cc}
1 & 0 & 0 & 1 & 1 & 0 \\
0 & 0 & 0 & 0 & 0 & 0 \\
0 & 1 & \star & \star & 0 & 1 \\
0 & 0 & 1 & 0 & \star & \star
\end{array}\right) \\
\mathcal{I}=(13,14,14) \\
\mathcal{I}=(13,34,13)
\end{gathered}
$$

The product of the affine paving of $\bigsqcup_{\mathcal{I}}$ is $\mathcal{J}$-compatible $D_{\mathcal{I}}$ shown above with the cell $\Omega_{\mathcal{J}}$ gives the desired paving of $\Omega_{\mathcal{J}}$ by the cells $C_{\mathcal{I}}$ by means of (4.21). 


\section{Cohomology presentation}

5.1. $\mathbb{Z}$-modules. We aim to show that $H^{\bullet}\left(X_{\alpha, k} ; \mathbb{Z}\right)$ is the $\mathfrak{S}_{\alpha}$-invariant subring $\left(R_{\alpha, k}\right)^{\mathfrak{S}_{\alpha}}$ of $R_{\alpha, k}$. Theorem 3.10 gives information about the rational version $S_{\alpha, k}=\mathbb{Q} \otimes_{\mathbb{Z}} R_{\alpha, k}$ of $R_{\alpha, k}$. We will need to relate vector spaces over $\mathbb{Q}$ and modules over $\mathbb{Z}$. We collect several basic facts in this direction here. Recall that a $\mathbb{Z}$-module $M$ is free of rank $r$ if $M$ has an $r$-element $\mathbb{Z}$-basis.

Lemma 5.1. (1) Let $M$ be a free $\mathbb{Z}$-module of finite rank, so that $\mathbb{Q} \otimes_{\mathbb{Z}} M$ is a $\mathbb{Q}$-vector space. We have $\operatorname{dim}\left(\mathbb{Q} \otimes_{\mathbb{Z}} M\right)=\operatorname{rank}(M)$.

(2) Let $M$ be a free $\mathbb{Z}$-module of finite rank and let $N \subseteq M$ be a submodule. Then $N$ is also a free $\mathbb{Z}$-module.

(3) Let $f: M \rightarrow N$ be a surjective homomorphism between free $\mathbb{Z}$-modules of finite rank. If $\operatorname{rank}(M)=\operatorname{rank}(N)$ then $f$ is an isomorphism.

Let $X$ be a complex algebraic variety and let $V$ be a vector bundle over $X$. Let $X^{\prime} \rightarrow X$ be the bundle over $X$ whose fiber over $x \in X$ is the space of complete flags in $V_{x}$. To present the integral cohomology $H^{\bullet}\left(X_{\alpha, k} ; \mathbb{Z}\right)$, we will need some facts about the embedding $H^{\bullet}(X ; \mathbb{Z}) \hookrightarrow H^{\bullet}\left(X^{\prime} ; \mathbb{Z}\right)$.

The bundle $X^{\prime} \rightarrow X$ is a sequence of projective bundles. Let $V \rightarrow X$ be a vector bundle of rank $r$ and let $p: \mathbb{P}(V) \rightarrow X$ be the associated projective bundle. Then the induced map $p^{*}: H^{\bullet}(X ; \mathbb{Z}) \rightarrow H^{\bullet}(\mathbb{P}(V) ; \mathbb{Z})$ is injective. In fact, we may present $H^{\bullet}(\mathbb{P}(V) ; \mathbb{Z})$ as

$$
H^{\bullet}(\mathbb{P}(V) ; \mathbb{Z})=H^{\bullet}(X ; \mathbb{Z})[\zeta] /\left\langle\zeta^{r}+c_{1}(V) \zeta^{r-1}+\cdots+c_{r}(V)\right\rangle,
$$

where the $c_{i}(V) \in H^{\bullet}(X ; \mathbb{Z})$ are the Chern classes of $V$ and $\zeta \in H^{2}(\mathbb{P}(V) ; \mathbb{Z})$ is the first Chern class of the tautological line bundle $\mathcal{O}(1)$ on $\mathbb{P}(V)$.

In our setting, the cohomology $H^{\bullet}(X ; \mathbb{Z})$ will be a free $\mathbb{Z}$-module of finite rank. The above discussion motivates the following lemma.

Lemma 5.2. Let $A$ be a free $\mathbb{Z}$-module of finite rank and let $t$ be a variable. Fix elements $a_{1}, a_{2}, \ldots, a_{r} \in A$ and consider the quotient

$$
B:=A[t] /\left\langle t^{r}+a_{1} t^{r-1}+\cdots+a_{r-1} t+a_{r}\right\rangle .
$$

We have the following.

(1) The ring $B$ is a free $\mathbb{Z}$-module.

(2) The natural map $A \rightarrow B$ is injective so we may view $A$ as a subset of $B$.

(3) For any integer $N>0$ and any $b \in B$ such that $N \cdot b \in A$ we have $b \in A$.

Proof. If $\alpha_{1}, \ldots, \alpha_{m}$ is a $\mathbb{Z}$-basis of $A$, it follows that $\left\{\alpha_{i} t^{j}: 1 \leq i \leq m, 0 \leq j \leq r-1\right\}$ is a $\mathbb{Z}$-basis of $B$, which proves (1). Statements (2) and (3) follow from the form of this basis.

5.2. The ring $H^{\bullet}\left(X_{\alpha, k} ; \mathbb{Z}\right)$. As a first application of Lemma 5.1, we have the following.

Lemma 5.3. Let $\alpha=\left(\alpha_{1}, \ldots, \alpha_{r}\right) \in[k]^{r}$ with $|\alpha|=n$.

(1) The ring $R_{\alpha, k}$ is a free $\mathbb{Z}$-module of rank $\left|\mathcal{O P} \mathcal{P}_{\alpha, k}\right|$.

(2) The invariant subring $\left(R_{\alpha, k}\right)^{\mathfrak{S}_{\alpha}}$ is a free $\mathbb{Z}$-module of rank $\left|\mathcal{O} \mathcal{P}_{\alpha, k}\right| /\left(\alpha_{1} ! \cdots \alpha_{r} !\right)$.

Proof. (1) We show that the collection $\mathcal{M}_{\alpha, k}$ of $\alpha$-nonskip monomials descends to a $\mathbb{Z}$-basis of $R_{\alpha, k}$. By Lemma 3.9 we have $\left|\mathcal{M}_{\alpha, k}\right|=\left|\mathcal{O} \mathcal{P}_{\alpha, k}\right|$, so would prove the claim. By Theorem 3.10, we know that these monomials descend to a $\mathbb{Q}$-basis of $S_{\alpha, k}=\mathbb{Q} \otimes_{\mathbb{Z}} R_{\alpha, k}$, so it suffices to prove that these monomials span $R_{\alpha, k}$ over $\mathbb{Z}$.

Let $m$ be any monomial in $\mathbb{Z}\left[\mathbf{x}_{n}\right]$. We need to show that $m$ lies in the span of $\mathcal{M}_{\alpha, k}$ modulo $I_{\alpha, k}$. If $m \in \mathcal{M}_{\alpha, k}$ this is clear, so assume that $m \notin \mathcal{M}_{\alpha, k}$. The last sentence of Proposition 3.11 implies that there is a nonzero polynomial $f \in I_{\alpha, k}$ (either a Demazure character or a complete homogeneous symmetric polynomial) such that $\operatorname{in}_{<}(f) \mid m$ with respect to the neglex term order 
and that the neglex-leading coefficient of $f$ equals 1 . If we let $m^{\prime}$ be the quotient $m^{\prime}:=m / \operatorname{in}_{<}(f)$, the neglex-leading term of $m^{\prime} \cdot f \in I_{\alpha, k}$ is $m$. Since $m^{\prime} \cdot f \equiv 0$ modulo $I_{\alpha, k}$, we get

$$
m \cong \text { a } \mathbb{Z} \text {-linear combination of monomials }<m \text { in neglex. }
$$

We are done by induction on the neglex order.

(2) By Lemma $5.1(2)$, we know that $\left(R_{\alpha, k}\right)^{\mathfrak{S}_{\alpha}}$ is a free $\mathbb{Z}$-module. By Theorem 3.10, we have $S_{\alpha, k} \cong \mathbb{Q}\left[\mathcal{O P} \mathcal{P}_{\alpha, k}\right]$ as $\mathbb{Q}\left[\mathfrak{S}_{\alpha}\right]$-modules. Since the action of $\mathfrak{S}_{\alpha}$ on $\mathcal{O} \mathcal{P}_{\alpha, k}$ is free, taking $\mathfrak{S}_{\alpha}$-invariants and applying Lemma 5.1 (1) gives

$$
\operatorname{rank}\left(R_{\alpha, k}\right)^{\mathfrak{S}_{\alpha}}=\operatorname{dim} \mathbb{Q} \otimes_{\mathbb{Z}}\left(R_{\alpha, k}\right)^{\mathfrak{S}_{\alpha}}=\operatorname{dim} S_{\alpha, k}^{\mathfrak{S}_{\alpha}}=\operatorname{dim} \mathbb{Q}\left[\mathcal{O P} \mathcal{P}_{\alpha, k}\right]^{\mathfrak{S}_{\alpha}}=\left|\mathcal{O} \mathcal{P}_{\alpha, k}\right| /\left|\mathfrak{S}_{\alpha}\right|,
$$

as desired.

We are ready to prove Theorem 1.4 and present the cohomology of $X_{\alpha, k}$. Let us recall the statement of the result.

Theorem 1.4. Let $\alpha=\left(\alpha_{1}, \ldots, \alpha_{r}\right) \in[k]^{r}$ be a sequence of positive integers with $\alpha_{1}+\cdots+\alpha_{r}=n$. The singular cohomology ring $H^{\bullet}\left(X_{\alpha, k} ; \mathbb{Z}\right)$ may be presented as

$$
H^{\bullet}\left(X_{\alpha, k} ; \mathbb{Z}\right)=\left(\mathbb{Z}\left[\mathbf{x}_{n}\right] / I_{\alpha, k}\right)^{\mathfrak{S}_{\alpha}}=\left(R_{\alpha, k}\right)^{\mathfrak{S}_{\alpha}},
$$

where $I_{\alpha, k} \subseteq \mathbb{Z}\left[\mathbf{x}_{n}\right]$ is the ideal generated by the complete homogeneous symmetric polynomials $h_{d}\left(\mathbf{x}_{n}^{(i)}\right)$ where $d>n-\alpha_{i}$ and $1 \leq i \leq r$ together with the elementary symmetric polynomials $e_{n}\left(\mathbf{x}_{n}\right), e_{n-1}\left(\mathbf{x}_{n}\right), \ldots, e_{n-k+1}\left(\mathbf{x}_{n}\right)$. Here the variables $\mathbf{x}_{n}^{(i)}$ represent the Chern roots of the tautological vector bundle $W_{i}^{*} \rightarrow X_{\alpha, k}$.

Proof. Theorem 4.12 implies that the inclusion $\iota: X_{\alpha, k} \hookrightarrow G r(\alpha, k)$ induces a surjective map

$$
\iota^{*}: H^{\bullet}(G r(\alpha, k) ; \mathbb{Z}) \rightarrow H^{\bullet}\left(X_{\alpha, k} ; \mathbb{Z}\right)
$$

on cohomology. It follows that $H^{\bullet}\left(X_{\alpha, k} ; \mathbb{Z}\right)$ is generated by the Chern classes of the bundles $W_{1}^{*}, \ldots, W_{r}^{*}$. In terms of the variables $x_{i}$, we get the following.

The cohomology ring $H^{\bullet}\left(X_{\alpha, k} ; \mathbb{Z}\right)$ is generated by the elementary symmetric polynomials in the partial variable sets

$$
e_{d}\left(\mathbf{x}_{n}^{(i)}\right), \quad 1 \leq i \leq r, 1 \leq d \leq \alpha_{i}
$$

Pushing forward relations under $\iota^{*}$, we have

$$
h_{d}\left(\mathbf{x}_{n}^{(i)}\right)=0, \quad 1 \leq i \leq r, k-\alpha_{i}+1 \leq d \leq k
$$

in $H^{\bullet}\left(X_{\alpha, k} ; \mathbb{Z}\right)$.

The Whitney Sum Formula provides additional relations in $H^{\bullet}\left(X_{\alpha, k} ; \mathbb{Z}\right)$. The addition map $\left(w_{1}, \ldots, w_{r}\right) \mapsto w_{1}+\cdots+w_{r}$ gives a vector bundle surjection

$$
W_{1} \oplus \cdots \oplus W_{r} \rightarrow \mathbb{C}^{k}
$$

where $\mathbb{C}^{k}$ is the trivial rank $k$ vector bundle over $X_{\alpha, k}$. This dualizes to give an injection

$$
\left(\mathbb{C}^{k}\right)^{*} \hookrightarrow W_{1}^{*} \oplus \cdots \oplus W_{r}^{*},
$$

so that the direct sum $W_{1}^{*} \oplus \cdots \oplus W_{r}^{*}$ has a trivial rank $k$ subbundle. If we let $U$ be the corresponding rank $n-k$ quotient of $W_{1}^{*} \oplus \cdots \oplus W_{r}^{*}$, the Whitney Sum Formula tells us that

$$
c_{\bullet}(U)=c_{\bullet}\left(W_{1}^{*}\right) \cdots c_{\bullet}\left(W_{r}^{*}\right)
$$

where

$$
c_{\bullet}\left(W_{i}^{*}\right)=\left(1+x_{\alpha_{1}+\cdots+\alpha_{i-1}+1} t\right)\left(1+x_{\alpha_{1}+\cdots+\alpha_{i-1}+2} t\right) \cdots\left(1+x_{\alpha_{1}+\cdots+\alpha_{i-1}+\alpha_{i}} t\right) .
$$

Since $c_{\bullet}(U)$ is a polynomial in $t$ of degree $\leq n-k$, we have $e_{d}\left(\mathbf{x}_{n}\right)=0$ in $H^{\bullet}\left(X_{\alpha, k} ; \mathbb{Z}\right)$ for each $d>n-k$. 
Let $p: X_{\alpha, k}^{\prime} \rightarrow X_{\alpha, k}$ be the bundle whose fiber over a point $\left(W_{1}, \ldots, W_{r}\right) \in X_{\alpha, k}$ is the product space $\mathcal{F} \ell\left(W_{1}\right) \times \cdots \times \mathcal{F} \ell\left(W_{r}\right)$ of complete flags in $W_{1}, \ldots, W_{r}$. The Chern roots $x_{1}, \ldots, x_{n}$ are elements of $H^{\bullet}\left(X_{\alpha, k}^{\prime} ; \mathbb{Z}\right)$. Any $\mathfrak{S}_{\alpha}$-invariant polynomial in these Chern roots lies in $H^{\bullet}\left(X_{\alpha, k} ; \mathbb{Z}\right)$. The map $p$ induces an inclusion $p^{*}: H^{\bullet}\left(X_{\alpha, k} ; \mathbb{Z}\right) \hookrightarrow H^{\bullet}\left(X_{\alpha}^{\prime}, \mathbb{Z}\right)$. The second sentence in italics and the paragraph above imply that we have a map of $\mathbb{Z}$-algebras

$$
\widehat{\varphi}:\left(R_{\alpha, k}\right)^{\mathfrak{S}_{\alpha}}=\left(\mathbb{Z}\left[\mathbf{x}_{n}\right] / I_{\alpha, k}\right)^{\mathfrak{S}_{\alpha}} \rightarrow H^{\bullet}\left(X_{\alpha, k}^{\prime} ; \mathbb{Z}\right) .
$$

We claim that the image of $\widehat{\varphi}$ in fact lies in the subring $H^{\bullet}\left(X_{\alpha, k} ; \mathbb{Z}\right)$ of $H^{\bullet}\left(X_{\alpha, k}^{\prime} ; \mathbb{Z}\right)$. If we were using rational coefficients, this would be shown as follows. Let $\alpha !:=\alpha_{1} ! \cdots \alpha_{r}$ ! be the size of the group $\mathfrak{S}_{\alpha}$. Given any $f \in\left(R_{\alpha, k}\right)^{\mathfrak{S}_{\alpha}}$ we could average $\widehat{\varphi}(f)$ over $\mathfrak{S}_{\alpha}$ to obtain

$$
\widehat{\varphi}(f)=\widehat{\varphi}\left(\frac{1}{\alpha !} \sum_{\pi \in \mathfrak{S}_{\alpha}} \pi \cdot f\right)=\frac{1}{\alpha !} \sum_{\pi \in \mathfrak{S}_{\alpha}} \pi \cdot \widehat{\varphi}(f),
$$

where the first equality would use the fact that $f$ is $\mathfrak{S}_{\alpha}$-invariant. We see that $\widehat{\varphi}(f)$ would also be also $\mathfrak{S}_{\alpha}$-invariant and would lie in the cohomology of $X_{\alpha, k}$.

Since we are using integer coefficients, we cannot divide by $\alpha$ ! and the argument in the above paragraph does not work. However, this argument can be salvaged. For $f \in\left(R_{\alpha, k}\right)^{\mathfrak{S}_{\alpha}}$ the image

$$
\alpha ! \cdot \widehat{\varphi}(f)=\widehat{\varphi}(\alpha ! \cdot f)=\widehat{\varphi}\left(\sum_{\pi \in \mathfrak{S}_{\alpha}} \pi \cdot f\right)=\sum_{\pi \in \mathfrak{S}_{\alpha}} \pi \cdot \widehat{\varphi}(f),
$$

of $\alpha$ ! $f$ under $\widehat{\varphi}$ is $\mathfrak{S}_{\alpha}$-invariant and so lies in $H^{\bullet}\left(X_{\alpha, k} ; \mathbb{Z}\right)$. We need to show that $\widehat{\varphi}(f)$ itself lies in $H^{\bullet}\left(X_{\alpha, k} ; \mathbb{Z}\right)$. Since $p: X_{\alpha, k}^{\prime} \rightarrow X_{\alpha, k}$ is a sequence of projective bundles and $H^{\bullet}\left(X_{\alpha, k} ; \mathbb{Z}\right)$ is a free $\mathbb{Z}$-module of finite rank, the ring extension $p^{*}: H^{\bullet}\left(X_{\alpha, k} ; \mathbb{Z}\right) \hookrightarrow H^{\bullet}\left(X_{\alpha, k}^{\prime} ; \mathbb{Z}\right)$ is a sequence of extensions of the form addressed in Lemma 5.2. By Lemma 5.2 (3) and induction, we have $\widehat{\varphi}(f) \in H^{\bullet}\left(X_{\alpha, k} ; \mathbb{Z}\right)$.

Since the image of $\widehat{\varphi}$ lies in $H^{\bullet}\left(X_{\alpha, k} ; \mathbb{Z}\right)$ we may restrict the codomain of $\widehat{\varphi}$ to get a map of $\mathbb{Z}$-algebras

$$
\varphi:\left(R_{\alpha, k}\right)^{\mathfrak{S}_{\alpha}}=\left(\mathbb{Z}\left[\mathbf{x}_{n}\right] / I_{\alpha, k}\right)^{\mathfrak{S}_{\alpha}} \rightarrow H^{\bullet}\left(X_{\alpha, k} ; \mathbb{Z}\right) .
$$

The first sentence in italics above implies that $\varphi$ is a surjection. By Lemma 5.3 and Theorem 4.12 (as well as Lemma 4.11), we know that the domain and codomain of $\varphi$ are free of rank $\left|\mathcal{O P} \mathcal{P}_{\alpha, k}\right| / \alpha$ !. By Lemma 5.1 (3) we conclude that $\varphi$ is an isomorphism.

Since the cohomology of $X_{\alpha, k}$ is concentrated in even degree, Theorem 1.4 and the Universal Coefficient Theorem imply that the rational cohomology of $X_{\alpha, k}$ is given by

$$
H^{\bullet}\left(X_{\alpha, k} ; \mathbb{Q}\right)=\mathbb{Q} \otimes_{\mathbb{Z}}\left(R_{\alpha, k}\right)^{\mathfrak{S}_{\alpha}}=\left(S_{\alpha, k}\right)^{\mathfrak{S}_{\alpha}} .
$$

The description of $H^{\bullet}\left(X_{\alpha, k} ; \mathbb{Z}\right)=\left(R^{\alpha}\right)^{\mathfrak{S}_{\alpha}}$ in terms of Chern roots implies the following result.

Corollary 5.4. Let $\alpha=\left(\alpha_{1}, \ldots, \alpha_{r}\right) \in[k]^{r}$ with $|\alpha|=n$. Let $\pi \in \mathfrak{S}_{r}$ and set $\pi . \alpha:=\left(\alpha_{\pi(1)}, \ldots, \alpha_{\pi(r)}\right)$. We have the following pair of commutative diagrams.
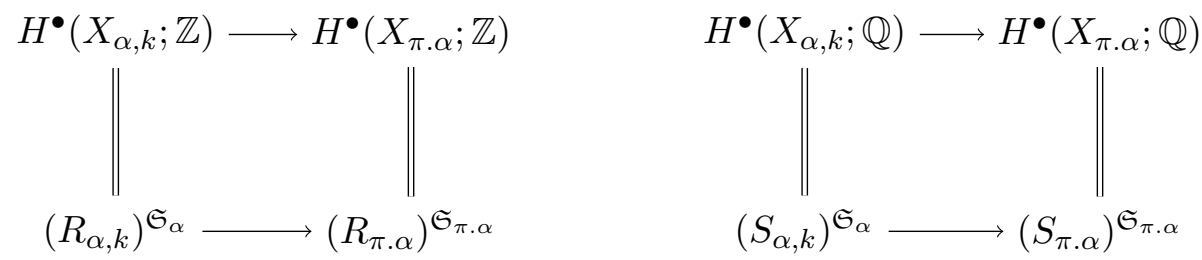

The vertical equalities come from Theorem 1.4 (and Equation (5.16)). The top horizontal arrows are induced from the action on subspaces $\left(W_{1}, \ldots, W_{r}\right) \mapsto\left(W_{\pi(1)}, \ldots, W_{\pi(r)}\right)$. The bottom horizontal arrows are induced by the action on batches of variables $\left(\mathbf{x}_{n}^{(1)}, \ldots, \mathbf{x}_{n}^{(r)}\right) \mapsto\left(\mathbf{x}_{n}^{(\pi(1))}, \ldots, \mathbf{x}_{n}^{(\pi(r))}\right)$. 
When the dimension vector $\alpha=(d, \ldots, d) \in[k]^{r}$ is constant, we have a combinatorial model for the ungraded representation $H^{\bullet}\left(X_{\alpha, k} ; \mathbb{Q}\right)$ of $\mathfrak{S}_{r}$.

Corollary 5.5. Let $\alpha=(d, \ldots, d) \in[k]^{r}$ be a constant dimension vector with $|\alpha|=r d=n$ so that $H^{\bullet}\left(X_{\alpha, k} ; \mathbb{Q}\right)$ is a $\mathfrak{S}_{r}$-module. Let $\mathcal{C}_{\alpha}$ be the family of set sequences $\mathcal{I}=\left(I_{1}, \ldots, I_{r}\right)$ in $[k]$ of type $\alpha$ which cover $[k]$.

The symmetric group $\mathfrak{S}_{r}$ acts on $\mathcal{C}_{\alpha}$ by subscript permutation, viz. $\pi .\left(I_{1}, \ldots, I_{r}\right):=\left(I_{\pi(1)}, \ldots, I_{\pi(r)}\right)$. We have an isomorphism of ungraded $\mathfrak{S}_{r}$-modules

$$
H^{\bullet}\left(X_{\alpha, k} ; \mathbb{Q}\right) \cong \mathbb{Q}\left[\mathcal{C}_{\alpha}\right] .
$$

Proof. The wreath product $\mathfrak{S}_{d} \prec \mathfrak{S}_{r}$ is the subgroup of $\mathfrak{S}_{n}$ given by the semidirect product

$$
\mathfrak{S}_{d} \prec \mathfrak{S}_{r}:=\mathfrak{S}_{r} \rtimes\left(\mathfrak{S}_{d} \times \cdots \times \mathfrak{S}_{d}\right)=\mathfrak{S}_{r} \rtimes \mathfrak{S}_{\alpha},
$$

where there are $r$ factors of $\mathfrak{S}_{d}$. The symmetric group $\mathfrak{S}_{r}$ acts on the $r$-fold product $\mathfrak{S}_{\alpha}$ by factor permutation. We have a natural embedding

$$
\mathfrak{S}_{\alpha}=1 \rtimes \mathfrak{S}_{\alpha} \subseteq \mathfrak{S}_{r} \rtimes \mathfrak{S}_{\alpha}=\mathfrak{S}_{d} \succ \mathfrak{S}_{r} .
$$

The point locus $Z_{\alpha, k} \subseteq \mathbb{Q}^{n}$ of Definition 3.3 is closed under the action of $\mathfrak{S}_{d} \succ \mathfrak{S}_{r}$. Theorem 3.10 (and its proof) give an isomorphism of ungraded $\mathfrak{S}_{d} \prec \mathfrak{S}_{r}$-modules $S_{\alpha, k} \cong \mathbb{Q}\left[\mathcal{O} \mathcal{P}_{\alpha, k}\right]$. Taking $\mathfrak{S}_{\alpha^{-}}$ invariants and looking and the residual action of $\mathfrak{S}_{r}$ gives the corollary.

\section{Open Problems}

For any $\alpha \in[k]^{r}$ with $|\alpha|=n$, Theorem 1.4 gives a geometric model for the ring of $\mathfrak{S}_{\alpha}$-invariants $\left(R_{\alpha, k}\right)^{\mathfrak{S}_{\alpha}}$. It is natural to ask for a geometric model for $R_{\alpha, k}$ itself. One candidate is as follows.

Definition 6.1. Let $\alpha=\left(\alpha_{1}, \ldots, \alpha_{r}\right) \in[k]^{r}$ with $|\alpha|=n$. Let $Y_{\alpha, k}$ be the moduli space of $n$-tuples $\left(\ell_{1}, \ldots, \ell_{n}\right)$ of 1 -dimensional subspaces $\ell_{i} \subseteq \mathbb{C}^{k}$ such that

- we have $\ell_{1}+\cdots+\ell_{n}=\mathbb{C}^{k}$ and

- for each $1 \leq i \leq r$, the subspace $\ell_{\alpha_{1}+\cdots+\alpha_{i-1}+1}+\cdots+\ell_{\alpha_{1}+\cdots+\alpha_{i-1}+\alpha_{i}}$ of $\mathbb{C}^{k}$ spanned by the $i^{\text {th }}$ batch of lines is $\alpha_{i}$-dimensional.

Wilson and the author [10] studied $Y_{\alpha, k}$ in the special case $\alpha=(m, 1, \ldots, 1)$.

Conjecture 6.2. We have the presentation $H^{\bullet}\left(Y_{\alpha, k} ; \mathbb{Z}\right)=R_{\alpha, k}$ where the variable $x_{i}$ represents the Chern class $c_{1}\left(\ell_{i}^{*}\right)$ of the line bundle $\ell_{i}^{*} \rightarrow Y_{\alpha, k}$.

Conjecture 6.2 is supported by the following fact about rational cohomology. There is a natural projection

$$
\pi: Y_{\alpha, k} \rightarrow X_{\alpha, k}
$$

which sends the line tuple $\left(\ell_{1}, \ldots, \ell_{n}\right)$ to the subspace tuple $\left(W_{1}, \ldots, W_{r}\right)$ where

$$
W_{i}=\ell_{\alpha_{1}+\cdots+\alpha_{i-1}+1}+\cdots+\ell_{\alpha_{1}+\cdots+\alpha_{i-1}+\alpha_{i}}
$$

is the span of the $i^{\text {th }}$ batch of lines.

The map $\pi$ is a fiber bundle with fiber $F$ homotopy equivalent to the product

$$
\mathcal{F} \ell\left(\alpha_{1}\right) \times \cdots \times \mathcal{F} \ell\left(\alpha_{r}\right)
$$

of flag varieties. The inclusion $\iota: F \hookrightarrow Y_{\alpha, k}$ endows $H^{\bullet}(F ; \mathbb{Q})$ induces a map $\iota^{*}: H^{\bullet}\left(Y_{\alpha, k} ; \mathbb{Q}\right) \rightarrow$ $H^{\bullet}(F ; \mathbb{Q})$ on rational cohomology.

Let $\alpha \in[k]^{r}$ with $|\alpha|=n$. Theorem 4.12 and Lemma 4.11 imply that the cohomology ring $H^{\bullet}\left(X_{\alpha, k} ; \mathbb{Z}\right)$ has $\mathbb{Z}$-basis given by the classes $\left\{\left[\overline{C_{\mathcal{I}}}\right]: \mathcal{I}\right.$ covers $\left.[k]\right\}$ of cell closures corresponding to covering set sequences $\mathcal{I}=\left(I_{1}, \ldots, I_{r}\right)$ in $[k]$ of type $\alpha$. In light of Theorem 1.4, it is natural to pose the following problem. 
Problem 6.3. Given a covering set sequence $\mathcal{I}=\left(I_{1}, \ldots, I_{r}\right)$ in $[k]$ of type $\alpha$, let $C_{\mathcal{I}} \subseteq X_{\alpha, k}$ be the corresponding cell. Find a polynomial in $\mathbb{Z}\left[\mathbf{x}_{n}\right]$ which represents $\left[\overline{C_{\mathcal{I}}}\right]$ in $H^{\bullet}\left(X_{\alpha, k} ; \mathbb{Z}\right)=\left(R_{\alpha, k}\right)^{\mathfrak{S}_{\alpha}}$.

When $k=r=n$, the set sequences $\mathcal{I}$ appearing in Problem 6.3 may be identified with permutations in $\mathfrak{S}_{n}$ and the classical Schubert polynomials solve Problem 6.3. When $k \leq r=n$, a solution to Problem 6.3 is given by the 'Fubini word Schubert polynomials' found in 8 .

Assuming the map $\iota^{*}$ is surjective, the Leray-Hirsch Theorem would give the following isomorphism of $H^{\bullet}\left(X_{\alpha, k} ; \mathbb{Q}\right)$-modules:

$$
H^{\bullet}\left(Y_{\alpha, k} ; \mathbb{Q}\right) \cong H^{\bullet}(F ; \mathbb{Q}) \otimes_{\mathbb{Q}} H^{\bullet}\left(X_{\alpha, k} ; \mathbb{Q}\right)=H^{\bullet}(F ; \mathbb{Q}) \otimes\left(S_{\alpha, k}\right)^{\mathfrak{S}_{\alpha}} .
$$

The surjectivity of $\iota^{*}$ would likely follow by constructing an affine paving of the $n$-fold product of $k-1$-dimensional complex projective spaces $\left(\mathbb{P}^{k-1}\right)^{n}$ whose initial part gives an affine paving of $Y_{\alpha, k} \subseteq\left(\mathbb{P}^{k-1}\right)^{n}$. Such a paving was constructed in the case $\alpha=(m, 1, \ldots, 1)$ in [10]. By the Künneth Theorem, the ring $H^{\bullet}(F ; \mathbb{Q})$ is isomorphic to a direct product of classical coinvariant rings, so that the isomorphism (6.3) would prove Conjecture 6.2 at the level of graded $\mathbb{Q}$-vector spaces.

Assume that the dimension vector $\alpha=(d, \ldots, d) \in[k]^{r}$ is constant. Corollary 5.5 describes the cohomology ring $H^{\bullet}\left(X_{\alpha, k} ; \mathbb{Q}\right)=\left(S_{\alpha, k}\right)^{\mathfrak{S}_{\alpha}}$ as an ungraded $\mathbb{Q}\left[\mathfrak{S}_{r}\right]$-module. This suggests the following problem.

Problem 6.4. Let $\alpha=(d, \ldots, d) \in[k]^{r}$ be a constant dimension vector.

- Determine the isomorphism type of $\left(S_{\alpha, k}\right)^{\mathfrak{S}_{\alpha}}$ as a graded $\mathfrak{S}_{r}$-module.

- Determine the isomorphism type of $S_{\alpha, k}$ as a graded $\mathfrak{S}_{d} \prec \mathfrak{S}_{r}$-module.

In the case $\alpha=(1, \ldots, 1)$, the two bullet points of Problem 6.4 coincide and $S_{\alpha, k}$ gives an algebraic and geometric model for the Delta Conjecture at $t=0$ [7, 8. A solution to Problem 6.4 could suggest an extension of the Delta Conjecture itself to general dimension vectors $\alpha$.

Borel's presentation of the partial flag variety $\mathcal{F} \ell(\alpha)$ in Theorem 1.3 has a generalization to arbitrary Lie type. In particular, if $G$ is a reductive complex Lie group and if $P$ is a parabolic subgroup of $G$, the homogeneous space $G / P$ has rational cohomology presented in terms of the coordinate ring of the Cartan subalgebra $\mathfrak{h}$ of $G$. It would be interesting to extend our results on the more general spaces $X_{\alpha, k}$ to arbitrary Lie type.

\section{AcKnowledgements}

The author is grateful to Sara Billey, Brendan Pawlowski, Vic Reiner, and Andy Wilson for many helpful conversations. The author thanks François Bergeron for asking how to generalize $X_{n, k}$ to higher-dimensional subspaces. The author was partially supported by NSF Grant DMS-1500838.

\section{REFERENCES}

[1] F. Bergeron. Algebraic Combinatorics and Coinvariant Spaces. CMS Treatises in Mathematics. Canadian Mathematical Society. 2009.

[2] A. Borel. Sur la cohomologie des espaces fibrés principaux et des espaces homogènes de groupes de Lie compacts. Ann. of Math. 57 (1953) 115-207.

[3] W. Fulton. Young Tableaux. London Mathematical Society Student Texts No. 35. Cambridge University Press. 1997.

[4] A. Garsia, J. Haglund, J. Remmel, and M. Yoo. A proof of the Delta Conjecture when $q=0$. Preprint, 2017. arXiv: 1710.07078 .

[5] J. Haglund, K. Luoto, S. Mason, and S. van Willigenburg. Refinements of the Littlewood-Richardson Rule. Trans. Amer. Math. Soc., 363 (2011), 1665-1686.

[6] J. Haglund, J. Remmel, and A. T. Wilson. The Delta Conjecture. Trans. Amer. Math. Soc., 370 (2018), 40294057.

[7] J. Haglund, B. Rhoades, and M. Shimozono. Ordered set partitions, generalized coinvariant algebras, and the Delta Conjecture. Adv. Math., 329 (2018), 851-915. 
[8] B. Pawlowski and B. Rhoades. A flag variety for the Delta Conjecture. Accepted, Trans. Amer. Math. Soc., 2019. arXiv: 1711.08301

[9] B. Rhoades. Ordered set partition statistics and the Delta Conjecture. J. Combin. Theory Ser. A 154 (2018), $172-217$.

[10] B. Rhoades and A. T. Wilson. Line configurations and $r$-Stirling partitions. J. Comb., 10 (3) (2019), $411-431$.

[11] A. T. Wilson. An extension of MacMahon's Equidistribution Theorem to ordered multiset partitions. Electron. J. Combin. 23.1 (2016). P1.5.

DePartment of Mathematics

University of CALifornia, SAN Diego

LA Jolla, CA, 92093-0112, USA

E-mail address: bprhoades@math.ucsd.edu 